EPJ manuscript No.

(will be inserted by the editor)

\title{
A gauge invariant chiral unitary framework for kaon photo- and electroproduction on the proton
}

\author{
B. Borasoy ${ }^{1}$, P. C. Bruns ${ }^{1}$, U.-G. Meißner ${ }^{1,2}$ and R. Nißler ${ }^{1}$ \\ 1 Helmholtz-Institut für Strahlen- und Kernphysik (Theorie) Universität Bonn, Nußallee 14-16, D-53115 Bonn, Germany \\ 2 Institut für Kernphysik (Theorie), Forschungszentrum Jülich D-52425 Jülich, Germany
}

Received: date / Revised version: date

\begin{abstract}
We present a gauge invariant approach to photoproduction of mesons on nucleons within a chiral unitary framework. The interaction kernel for meson-baryon scattering is derived from the chiral effective Lagrangian and iterated in a Bethe-Salpeter equation. Within the leading order approximation to the interaction kernel, data on kaon photoproduction from SAPHIR, CLAS and CBELSA/TAPS are analyzed in the threshold region. The importance of gauge invariance and the precision of various approximations in the interaction kernel utilized in earlier works are discussed.
\end{abstract}

PACS. 13.60.Le Meson production - 25.20.Lj Photoproduction reactions - 12.39.Fe Chiral Lagrangians

\section{Introduction}

The hadronic spectrum is still the least understood property of QCD. Most theoretical models predict much more states than are actually observed so far in experiments, see e.g. [1-3]. This is sometimes called the 'missing resonance problem'. The search for missing resonances has therefore been an important goal of various experimental efforts. The properties of baryon resonances are presently under thorough investigation at several facilities, e.g., at ELSA, JLab, MAMI GRAAL, COSY and SPring-8. Due to their hadronic decay modes, however, many baryon resonances have large overlapping widths which makes it a difficult task to study individual states. In this respect, polarization observables can be used as a tool to filter out specific resonances in specific reactions.

A possible explanation of the missing resonances could be that these states do not couple strongly to the pionnucleon channels which have provided to a large extent the resonance data. A strong coupling of these resonances to channels with strange particles could be unraveled in photoproduction processes of $K \Lambda$ and $K \Sigma$. Such experiments have been recently undertaken at SAPHIR $[4,5]$ and CLAS $[6,7]$ with high precision. More recently, $K^{0} \Sigma^{+}$has been measured with the CB/TAPS detector at ELSA [8]. These data indicate that resonances so far undetected might have been observed, but an unambiguous theoretical interpretation is still lacking. In addition, the beam polarization asymmetry for $\gamma p \rightarrow K^{+} \Lambda, K^{+} \Sigma^{0}$ has been investigated by the LEPS collaboration at SPring-8 [9].

Based on an analysis of cross sections, beam asymmetries, and recoil polarizations, the Bonn-Gatchina resonance model, e.g., demands among others the $P_{11}(1840)$,
$D_{13}(1870), D_{13}(2170)$ resonances $[10,11]$, whereas in the model of [12] a $P_{13}(1830)$ is preferred.

Electroproduction experiments are even more sensitive to the structure of the nucleon due to the longitudinal coupling of the virtual photon to the nucleon spin and might in addition yield some insight into the possible onset of perturbative QCD, see e.g. $[13,14]$. But at low photon virtuality experimental data for $\gamma p \rightarrow K \Lambda, K \Sigma$ are still not available.

Apparently, there are sufficient data to be met by theoretical approaches. In addition to the photoproduction data, further experimental constraints are provided by pion-induced reactions on the proton. Any theoretical approach that aims to describe the photoproduction data, ought to be consistent with the corresponding pion-induced data.

A successful theoretical approach to meson-baryon scattering is provided by chiral unitary methods, see e.g. [1520]. In this framework the chiral effective Lagrangian is utilized to derive, for example, the interaction kernel in a Bethe-Salpeter equation (BSE) which iterates mesonbaryon rescattering to infinite order. The BSE generates resonances dynamically, hence, without their explicit inclusion the importance of resonances can be studied. Chiral unitary approaches have been implemented quite successfully for photoproduction processes, see e.g. refs. [21$24]$, but as a simplification only those diagrams were taken into account where the photon is absorbed first and then the produced meson-baryon pair undergoes final state interaction. This simplified treatment violates, in general, gauge invariance. In order to guarantee gauge invariance, diagrams with the photon coupling to any intermediate state of the meson-baryon bubble chain must be taken into 
B. Borasoy et al.: A gauge invariant chiral unitary framework for kaon photo- and electroproduction on the proton

account $[25,26]$. One goal of the present work is to study the importance of these additional contributions which render the amplitude gauge invariant, but have been neglected in previous works.

Another simplification in chiral unitary approaches is the reduction of the interaction kernel to the on-shell point. Although the interaction kernel appears in loops, it has been argued that the off-shell components can be absorbed by redefining the coupling constants [27]. In this work, we do not employ the on-shell approximation but present two alternative methods to retain the off-shell components in the interaction kernel. For a discussion of these issues, we refer the reader to ref. [28].

In chiral unitary approaches to meson photoproduction, it has been common practice to utilize $s$-wave projections for the meson-baryon scattering kernel and the photoproduction multipoles. We will also analyze the accuracy of this approximation since in our approach some higher partial waves are generated through the small components of the Dirac spinors describing the baryon octet fields and through the kaon (baryon) pole term in charged (neutral) meson production.

The main goal of the present study is the construction of a minimal approach to meson photoproduction based on the chiral effective Lagrangian which is exactly unitary and gauge invariant. The presented method fulfills these important requirements from field theory, while at the same time any subset of diagrams cannot be omitted as this would violate unitarity or gauge invariance. In this study, we restrict ourselves to the chiral effective Lagrangian at leading order. The inclusion of higher chiral orders in the interaction kernel is straightforward and necessary to obtain better agreement with experiment, particularly at higher energies away from the respective thresholds. This will be the subject of forthcoming work.

The manuscript is organized as follows. In the next section, the effective Lagrangian and the Bethe-Salpeter formalism including off-shell components are introduced. The gauge invariant extension to photo- and electroproduction processes is discussed in sect. 3. Section 4 contains the comparison with experimental data on kaon photoproduction. The phenomenological impact of the violation of gauge invariance and the use of the on-shell approximation is also discussed. We summarize our findings in sect. 5] while lengthy formulae and a second, alternative method for including off-shell pieces in the interaction kernel are relegated to the appendices.

\section{Bethe-Salpeter equation}

The chiral effective Lagrangian incorporates symmetries and symmetry-breaking patterns of QCD in a model-independent way, in particular chiral symmetry and its explicit breaking through the finite quark masses. By expanding Green's functions in powers of Goldstone boson masses and small momenta a chiral counting scheme can be established. However, the strict perturbative chiral expansion is only applicable at low energies, and it certainly fails in the vicinity of resonances. In this respect, the combination of the chiral effective Lagrangian with nonperturbative schemes based on coupled channels and the Bethe-Salpeter equation (BSE) have proven useful both in the purely mesonic and in the meson-baryon sector [15-20]. Such approaches extend the range of applicability of the chiral effective Lagrangian by implementing exact two-body unitarity in a non-perturbative fashion and generating resonances dynamically.

In this work we restrict ourselves to the meson-baryon Lagrangian at leading order

$$
\begin{aligned}
\mathcal{L}_{\phi B}^{(1)} & =\left\langle\bar{B}\left([i \not D, B]-m_{0} B\right)\right\rangle \\
& -\frac{D}{2}\left\langle\bar{B} \gamma^{\mu} \gamma_{5}\left\{u_{\mu}, B\right\}\right\rangle-\frac{F}{2}\left\langle\bar{B} \gamma^{\mu} \gamma_{5}\left[u_{\mu}, B\right]\right\rangle,
\end{aligned}
$$

where the matrix $B$ collects the ground state baryon octet, $\langle\ldots\rangle$ denotes the trace in flavor space, $m_{0}$ is the common baryon octet mass in the chiral limit and

$$
\left[D^{\mu}, B\right]=\partial^{\mu} B+\left[\Gamma^{\mu}, B\right]
$$

is the covariant derivative of the baryon field with the 'chiral connection'

$$
\Gamma^{\mu}=\frac{1}{2}\left[u^{\dagger}, \partial^{\mu} u\right]-\frac{i}{2}\left(u^{\dagger} v^{\mu} u+u v^{\mu} u^{\dagger}\right) .
$$

The external vector field is given by $v_{\mu}=-e Q \mathcal{A}_{\mu}$ where $Q=\frac{1}{3} \operatorname{diag}(2,-1,-1)$ is the quark charge matrix. The lowest lying octet of pseudoscalar meson fields $\phi$ enters the Lagrangian, in matrix form, as

$$
U(\phi)=u^{2}(\phi)=\exp \left(\sqrt{2} i \frac{\phi}{f_{0}}\right),
$$

where $f_{0}$ denotes the pseudoscalar decay constant in the chiral limit. Furthermore, we use

$$
\begin{aligned}
u_{\mu} & =i u^{\dagger} \nabla_{\mu} U u^{\dagger}, \\
\nabla_{\mu} U & =\partial_{\mu} U-i\left[v_{\mu}, U\right],
\end{aligned}
$$

and the coupling constants $D=0.8, F=0.46[29]$. By expanding the chiral connection in powers of the meson fields, one derives from the effective Lagrangian the leading order $\phi^{2} \bar{B} B$ vertex (the so-called 'Weinberg-Tomozawa' (WT) term), which we use as the driving term in our Bethe-Salpeter equation. Stated differently, this vertex insertion is the 'interaction kernel' of the integral equation. One finds for the corresponding potential $V$ (which is the WT-vertex graph multiplied by $i$ )

$$
V^{b j, a i}\left(\not_{2}, q_{1}\right)=g^{b j, a i}\left(q_{1}+\not q_{2}\right) \text {. }
$$

Here, $q_{1}$ and $q_{2}$ are the four-momenta of the incoming and the outgoing meson, respectively, and the coupling constants are summarized as a matrix in channel space, with the entries

$$
g^{b j, a i}=-\frac{1}{4 f_{0}^{2}}\left\langle\lambda^{b \dagger}\left[\left[\lambda^{j \dagger}, \lambda^{i}\right], \lambda^{a}\right]\right\rangle,
$$




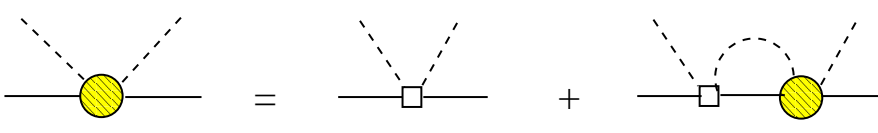

Fig. 1. Graphical illustration of the BSE for meson-baryon scattering. The filled circle represents the full scattering matrix and the open square the driving meson-baryon vertex.

where $\lambda^{a}$ are the generators of the $S U(3)$ Lie-Algebra in the physical (particle) basis. In this representation, a double index $b j$ specifies a particular channel consisting of a baryon $b$ and a meson $j$. The baryon and the meson propagator, $i S$ and $i \Delta$, are also summarized as (diagonal) matrices in channel space. They are given by

$$
\begin{aligned}
i S^{b j, a i}(\not p) & =\frac{i \delta^{b a} \delta^{j i}}{\not p-m_{a}}, \\
i \Delta^{b j, a i}(p) & =\frac{i \delta^{b a} \delta^{j i}}{p^{2}-M_{j}^{2}} .
\end{aligned}
$$

We can now write down the integral equation for the meson-baryon scattering amplitude $T^{b j, a i}$ in a rather compact form (suppressing the channel indices, but remembering the matrix character of the various amplitudes):

$$
\begin{aligned}
& T\left(\not q_{2}, \not q_{1} ; p\right)=V\left(\not q_{2}, \not q_{1}\right) \\
& \quad+\int \frac{d^{d} l}{(2 \pi)^{d}} V\left(\not q_{2}, l\right) i S(\not p-\not) \Delta(l) T\left(l, q_{1} ; p\right) .
\end{aligned}
$$

Here, $p \equiv p_{1}+q_{1}=p_{2}+q_{2}$ is the overall momentum, where $p_{1}$ and $p_{2}$ are the four momenta of the incoming and outgoing baryon, respectively. The BSE is illustrated in fig. 1. Note that we use dimensional regularization throughout. The solution of the BSE reads

$$
\begin{aligned}
& T\left(\not_{2}, \not q_{1} ; p\right)=W\left(\not_{2}, \not q_{1} ; p\right) \\
& \quad+W\left(q_{2}, \tilde{p} ; p\right) G(p)[1-W(\tilde{p}, \tilde{p} ; p) G(p)]^{-1} W\left(\tilde{p}, q_{1} ; p\right)
\end{aligned}
$$

with

$$
\begin{aligned}
W\left(q_{2}, \not q_{1} ; p\right)= & q_{2} g \frac{1}{1+I_{\mathrm{M}} g}+\frac{1}{1+g I_{\mathrm{M}}} g q_{1} \\
& -g \frac{1}{1+I_{\mathrm{M}} g} I_{\mathrm{M}}(\not p-m) \frac{1}{1+g I_{\mathrm{M}}} g .
\end{aligned}
$$

$W\left(q_{2}, \tilde{p} ; p\right)$ can be obtained from eq. (2.11) by replacing $\not q_{1} \rightarrow \not p-m \equiv \tilde{p}$, and so on, $m$ is the baryon mass matrix with entries

$$
m^{b j, a i}=\delta^{b a} \delta^{j i} m_{a},
$$

and the loop integrals are given by

$$
\begin{aligned}
I_{\mathrm{M}}^{b j, a i} & =\int \frac{d^{d} l}{(2 \pi)^{d}} i \Delta^{b j, a i}(l), \\
G^{b j, a i}(p) & =\int \frac{d^{d} l}{(2 \pi)^{d}} i[\Delta(l) S(\not p-l)]^{b j, a i} .
\end{aligned}
$$

Note that in eqs. (2.10) and (2.11), the symbol ' 1 ' represents the unit matrix in channel space, while matrixvalued denominators denote matrix inversion. In order to verify that the above expression for $T$ indeed solves the $\mathrm{BSE}$, one employs the relation

$$
\begin{aligned}
& \int \frac{d^{d} l}{(2 \pi)^{d}} V\left(\phi_{2}, \not\right) i S(\not p-\not) \Delta(l) W(\not l, \not q ; p) \\
& \quad=V\left(q_{2}, \tilde{p}\right) G(p) W(\tilde{p}, \not q ; p)+W\left(\not q_{2}, \not ; ; p\right)-V\left(q_{2}, \not q\right)
\end{aligned}
$$

for arbitrary $q$. Substituting the expression for $T$ in the BSE, eq. (2.9) and using the last relation, it is straightforward to confirm that $T$ solves the integral equation, as claimed. It is important to keep in mind that all the involved expressions are matrices in channel space, so e.g. $I_{\mathrm{M}}$ does in general not commute with the coupling matrix $g$. The on-shell substitutions

$$
\not q_{1,2} \rightarrow \not p_{1,2}+\not q_{1,2}-m=\not p-m \equiv \tilde{p}
$$

which occur in the above expressions, provide the connection to the alternative method of solving the BSE presented in App. A. The solution given there is completely equivalent to the one described above. Here, again, we note that $\tilde{p}$ is a matrix in channel space due to the matrix character of the baryon mass matrix $m$.

Putting the external momenta on their respective mass shells, the form of the solution $T$ of eq. (2.9) simplifies to

$$
T_{\text {on }}=[1-W G]^{-1} W,
$$

where we used the shorthand notation $W \equiv W(\tilde{p}, \tilde{p} ; p)$ and $G \equiv G(p)$. To obtain the complete on-shell mesonbaryon scattering amplitude, this expression has to be sandwiched between baryon spinors $\bar{u}\left(p_{2}\right) \ldots u\left(p_{1}\right)$. Neglecting the tadpole integrals $I_{\mathrm{M}}$ in $W$, one obtains from eq. (2.17)

$$
T_{\text {on }}^{\prime}=[1-V G]^{-1} V,
$$

which is the form for the scattering amplitude usually encountered when utilizing the 'on-shell-approximation' (which consists of setting also internal loop momenta in the potential $V$ on-shell) to arrive at a simplified version of the BSE. The integral equation eq. (2.9) then reduces to an algebraic equation that can be solved by matrix inversion to give an expression like $T_{\mathrm{on}}^{\prime}$ above. Retaining the off-shell pieces in the exact solution $T_{\text {on }}$ therefore corresponds to keeping the terms proportional to $I_{\mathrm{M}}$ in the expression for $W$.

In [27] it was argued that the on-shell reduction of the interaction kernel is justified as the off-shell pieces can be absorbed into the vertices. While this procedure simplifies the interaction kernel and the treatment of the BSE, it is in fact not necessary in order to solve the BSE as explained above. We choose not to discard the off-shell pieces and evaluate the diagrams in the way dictated by field theory, keeping in mind, however, that the off-shell parts are not uniquely fixed by any physical requirements and depend on the field parametrization [28]. Recall that eq. (2.4) is 
not the only possible choice for the parametrization of the meson fields, and that the off-shell amplitudes in ChPT depend on this choice.

Another important reason for taking the full off-shell dependence of the interaction kernel into account is that the implementation of the BSE in a gauge-invariant description of photoproduction processes is then straightforward. This is because we evaluate the occurring loop graphs according to the rules of field theory which also ensures that gauge symmetry can be incorporated in a standard way. This is not the case when utilizing the onshell approximation.

At present, our interaction kernel is restricted to the lowest order contact term derived from the effective mesonbaryon Lagrangian. The extension to more general vertex structures in the kernel is possible and will be discussed in future work. For now, it is not our aim to achieve perfect agreement with experimental data (in which case higherorder terms in the kernel would be indispensable), but to construct the simplest possible amplitude for kaon photoproduction that reconciles the framework of chiral unitary approaches with the fundamental principle of gauge invariance in a straightforward way.

For the sake of completeness, we add some comments on unitarity here. From the expression for $T_{\text {on }}$, eq. (2.17), one immediately confirms the condition for two-particle unitarity

$$
\operatorname{Im}\left(T_{\text {on }}^{-1}\right)=-\operatorname{Im}(G) .
$$

Our approach to photoproduction presented in the next section will be such that unitarity is also satisfied when the photon is coupled to the meson-baryon bubble chain summed up by means of the BSE, see also [25].

The form of the solution $T_{\text {on }}$ guarantees that the above unitarity statement holds. However, the careful reader might have noticed that the integrals $I_{\mathrm{M}}$ and $G(p)$ occurring in the amplitude are divergent for $d \rightarrow 4$ and that the introduction of appropriate counterterms might spoil the simple form of the solution given in eq. (2.17). To deal with this complication one can show by means of a rather lengthy calculation that the modification

$$
V \rightarrow V+\delta V \equiv V_{\delta},
$$

of the interaction kernel $\mathrm{V}$ in the BSE, where

$$
\begin{aligned}
& V_{\delta}\left(q_{2}, q_{1} ; p\right)=W_{\delta}\left(q_{2}, \not q_{1} ; p\right) \\
& -W_{\delta}\left(q_{2}, \tilde{p} ; p\right) \delta G(p)\left[1+W_{\delta}(\tilde{p}, \tilde{p} ; p) \delta G(p)\right]^{-1} W_{\delta}\left(\tilde{p}, q_{1} ; p\right)
\end{aligned}
$$

and

$$
\begin{aligned}
W_{\delta}\left(q_{2}, q_{1} ; p\right)= & q_{2} g \frac{1}{1-\delta I_{\mathrm{M}} g}+\frac{1}{1-g \delta I_{\mathrm{M}}} g q_{1} \\
& +g \frac{1}{1-\delta I_{\mathrm{M}} g} \delta I_{\mathrm{M}}(\not p-m) \frac{1}{1-g \delta I_{\mathrm{M}}} g
\end{aligned}
$$

leads to a new integral equation with a solution $T_{\delta}$. The solution $T_{\delta}$ is obtained from $T$ by replacing

$$
\begin{aligned}
G & \rightarrow G-\delta G \\
I_{\mathrm{M}} & \rightarrow I_{\mathrm{M}}-\delta I_{\mathrm{M}}
\end{aligned}
$$

in eqs. (2.10) and (2.11). One observes that $V_{\delta}$ has the same form as $T$, with $G$ and $I_{\mathrm{M}}$ replaced by $-\delta G$ and $-\delta I_{\mathrm{M}}$, respectively. The corrections $\delta G$ and $\delta I_{\mathrm{M}}$ have the form of polynomial terms which serve to absorb the divergences in the loop integrals. The divergences have thus been shifted from the loop integrals in $T$ to the new kernel. Of course, this is not a renormalization scheme in the usual sense, since the additional terms $\delta V$ in $V_{\delta}$ do not correspond exactly to counterterms derived from an effective Lagrangian which is obvious form the lack of crossing symmetry of the amplitude. It has already been noted in ref. [28] that the solution of the BSE can not be renormalized within a usual renormalization scheme. However, the foregoing discussion shows at least that altering the loop integrals appearing in the solution of the BSE by terms $\delta G$ and $\delta I_{\mathrm{M}}$ is equivalent to certain modifications of the potential. In practice, we merely omit the divergences in the loop integrals in our expression for $T$, which will therefore depend on the regularization scale $\mu$ showing up in the modified loop integrals.

This form of the off-shell BSE solution $T$ is not particularly convenient for the purpose of implementing it into the photoproduction amplitude. Therefore, we rewrite it by using the following decompositions:

$$
\begin{aligned}
G(p) & =G_{1}(p) \not p+G_{0}(p), \\
W(\tilde{p}, \tilde{p} ; p) & =W_{1}(p) \not p+W_{0}(p),
\end{aligned}
$$

where the matrices $G_{0,1}$ follow straightforwardly from the explicit expression for the loop integral $G$ which is given in eq. (B.11) of App. B and

$$
\begin{aligned}
W_{1}(p)= & g \frac{2+I_{\mathrm{M}} g}{\left[1+I_{\mathrm{M}} g\right]^{2}}, \\
W_{0}(p)= & g \frac{1}{1+I_{\mathrm{M}} g}\left(I_{\mathrm{M}} m\right) \frac{1}{1+g I_{\mathrm{M}}} g \\
& -m g \frac{1}{1+I_{\mathrm{M}} g}-\frac{1}{1+g I_{\mathrm{M}}} g m .
\end{aligned}
$$

Note that $G_{i}(p)$ and $W_{i}(p)$ are Lorentz scalars which depend only on the variable $p^{2}$. Using these expressions one can derive

$$
1-W(\tilde{p}, \tilde{p} ; p) G(p)=\tilde{W}_{1 \not p}+\tilde{W}_{0},
$$

with

$$
\begin{aligned}
& \tilde{W}_{1}=-\left(W_{1}(p) G_{0}(p)+W_{0}(p) G_{1}(p)\right), \\
& \tilde{W}_{0}=1-p^{2} W_{1}(p) G_{1}(p)-W_{0}(p) G_{0}(p) .
\end{aligned}
$$

The above results yield

$$
G(p)[1-W(\tilde{p}, \tilde{p} ; p) G(p)]^{-1}=\Omega_{1}(p) \not p+\Omega_{0}(p)
$$

with

$$
\begin{aligned}
\Omega_{1}(p)= & G_{0}(p)\left[p^{2} \tilde{W}_{1}-\tilde{W}_{0} \tilde{W}_{1}^{-1} \tilde{W}_{0}\right]^{-1} \\
& -G_{1}(p) \tilde{W}_{1}^{-1} \tilde{W}_{0}\left[p^{2} \tilde{W}_{1}-\tilde{W}_{0} \tilde{W}_{1}^{-1} \tilde{W}_{0}\right]^{-1} \\
\Omega_{0}(p)= & p^{2} G_{1}(p)\left[p^{2} \tilde{W}_{1}-\tilde{W}_{0} \tilde{W}_{1}^{-1} \tilde{W}_{0}\right]^{-1} \\
& -G_{0}(p) \tilde{W}_{1}^{-1} \tilde{W}_{0}\left[p^{2} \tilde{W}_{1}-\tilde{W}_{0} \tilde{W}_{1}^{-1} \tilde{W}_{0}\right]^{-1} .
\end{aligned}
$$


B. Borasoy et al.: A gauge invariant chiral unitary framework for kaon photo- and electroproduction on the proton

All these results can be combined to provide the decomposition of the off-shell BSE-solution into the following independent Clifford algebra structures:

$$
\begin{aligned}
T\left(q_{2}, q_{1} ; p\right)= & \not q_{2} \not p q_{1} T_{1}(p)+\not q_{2} q_{1} T_{2}(p)+\not p q_{1} T_{3}(p) \\
& +q_{2} \not p T_{4}(p)+q_{1} T_{5}(p)+q_{2} T_{6}(p) \\
& +\not p T_{7}(p)+T_{8}(p) .
\end{aligned}
$$

The scalar coefficient functions $T_{n}(p)$ read

$$
\begin{aligned}
T_{1}(p)= & L_{1} \Omega_{1}(p) L_{1} \\
T_{2}(p)= & L_{1} \Omega_{0}(p) L_{1} \\
T_{3}(p)= & {\left[L_{2} \Omega_{0}(p)+L_{3} \Omega_{1}(p)\right] L_{1}, } \\
T_{4}(p)= & T_{3}^{T}(p) \\
T_{5}(p)= & {\left[p^{2} L_{2} \Omega_{1}(p)+L_{3} \Omega_{0}(p)\right] L_{1}+L_{1}, } \\
T_{6}(p)= & T_{5}^{T}(p), \\
T_{7}(p)= & {\left[p^{2} L_{2} \Omega_{1}(p)+L_{3} \Omega_{0}(p)\right] L_{2} } \\
& +\left[L_{2} \Omega_{0}(p)+L_{3} \Omega_{1}(p)\right] L_{3}^{T}-g I_{\mathrm{M}} L_{2}, \\
T_{8}(p)= & p^{2}\left[L_{2} \Omega_{0}(p) L_{2}+L_{3} \Omega_{1}(p) L_{2}+L_{2} \Omega_{1}(p) L_{3}^{T}\right] \\
& +L_{3} \Omega_{0}(p) L_{3}^{T}-L_{3} I_{\mathrm{M}} g,
\end{aligned}
$$

where the superscript $T$ denotes transposition of channel indices and

$$
\begin{aligned}
L_{1} & =g \frac{1}{1+I_{\mathrm{M}} g}, \\
L_{2} & =\frac{1}{\left[1+g I_{\mathrm{M}}\right]^{2}} g, \\
L_{3} & =-\frac{1}{1+g I_{\mathrm{M}}} g m \frac{1}{1+I_{\mathrm{M}} g} .
\end{aligned}
$$

Note that $L_{1}^{T}=L_{1}$ and $L_{2}^{T}=L_{2}$, but in general $L_{3}^{T} \neq$ $L_{3}$. For external on-shell particles, we can make the usual substitutions

$$
\not q_{1}, \not q_{2} \rightarrow \not p-m \equiv \tilde{p}
$$

to arrive at the on-shell scattering amplitude

$$
T_{\mathrm{on}}=T_{\mathrm{on}}^{(1)} \not p+T_{\mathrm{on}}^{(0)}
$$

with

$$
\begin{aligned}
T_{\mathrm{on}}^{(1)}= & p^{2} T_{1}+m T_{1} m-m T_{2}-T_{2} m \\
& -T_{3} m-m T_{4}+T_{5}+T_{6}+T_{7}, \\
T_{\mathrm{on}}^{(0)}= & p^{2}\left(T_{2}+T_{3}+T_{4}-T_{1} m-m T_{1}\right)+m T_{2} m \\
& -T_{5} m-m T_{6}+T_{8} .
\end{aligned}
$$

The functions $T_{n}$ given in eq. (2.34) depend only on the variable $p^{2}$. They will enter the calculation of the various photo- and electroproduction amplitudes in the next section.

\section{Photo- and electroproduction}

The Bethe-Salpeter approach discussed in the last section (or the Lippmann-Schwinger equation in the nonrelativistic framework) can be implemented in electroproduction processes of mesons on the nucleon. In previous work, the electromagnetic meson production on the nucleon was calculated at tree level and the produced mesonbaryon pair was subject to final-state interactions $[16,24$, 30]. As the photon does not couple to all intermediate states of the meson-baryon bubble chain, gauge invariance is in general violated and must be restored via artificial manipulations. Therefore it seems desirable to develop a formalism which implements the principles of gauge invariance and unitarity in a most natural and straightforward manner. We follow here the path which we have already outlined in [25], in rather general terms, for the case of a photon coupling to a meson-baryon scattering amplitude. In this work, we shall be more explicit in evaluating the contributions to the various amplitudes in question. Our approach for constructing a unitary and gauge invariant electroproduction amplitude decomposes into two major steps:

(1) Fix the hadronic part of the amplitude by making use of a BSE to implement exact two-body unitarity.

(2) Couple the photon to the 'hadronic skeleton' constructed in step (1) wherever possible, i.e. to all external and internal lines describing the propagation of the involved particles as well as to (momentum-dependent) vertices.

The procedure of step (2), which is the most natural way to guarantee gauge invariance of the amplitude, leads to contributions that were usually not considered in chiral unitary approaches involving electromagnetic interactions. The importance of these additional contributions which render the electroproduction amplitude gauge invariant can also be quantified within the scheme utilized here. The coupling of the photon to internal lines in the bubble chain generated by the BSE leads to rather involved expressions, since the meson-baryon scattering amplitude $T$ appears twice in the corresponding amplitudes, as will be made more explicit later when we give the formal expressions for the various contributions.

Exact unitarity (in the subspace of meson-baryon states) is a fundamental principle satisfied by our approach. It is important to note that the procedure of step (2) above does not spoil the requirement of unitarity we built in by means of the BSE in step (1), as we will show below. First, we have to specify the set of graphs which constitute our amplitude.

We start with the tree level $\bar{B} \phi B$ amplitude derived from the leading order Lagrangian of eq. (2.1),

$$
\hat{V}^{b i, a}=\not 1 \gamma_{5} \hat{g}^{b i, a}
$$

The last index $a$ labels the incoming baryon (here always the proton), while the double index belongs to the outgoing pair of baryon $b$ and meson $i$. For fixed $a, \hat{V}$ is a vector 


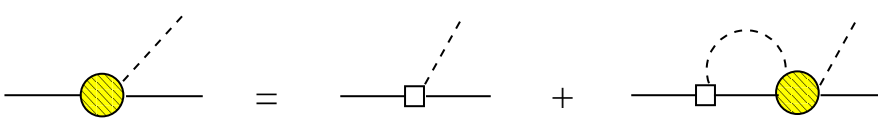

Fig. 2. The dressed meson-baryon vertex in the turtle approximation. The filled circle (open square) denotes the full (tree level) meson-baryon interaction.

in channel space. Furthermore, $q$ is the four-momentum of the outgoing meson and

$$
\hat{g}^{b i, a}=-\frac{D}{\sqrt{2} f_{0}}\left\langle\lambda^{b \dagger}\left\{\lambda^{i \dagger}, \lambda^{a}\right\}\right\rangle-\frac{F}{\sqrt{2} f_{0}}\left\langle\lambda^{b \dagger}\left[\lambda^{i \dagger}, \lambda^{a}\right]\right\rangle .
$$

To this tree level amplitude, we add the loop contribution that accounts for the final-state interaction after the meson has left the vertex, to obtain (cf. fig. 2)

$$
\Gamma(\not q, \not p)=\not q \gamma_{5} \hat{g}+\int \frac{d^{d} l}{(2 \pi)^{d}} T(\not q, l ; p) i S(\not p-\not) \Delta(l) l \gamma_{5} \hat{g} .
$$

We call this approximation to the full meson-baryon interaction the 'turtle approximation', because with some imagination the right-most diagram in fig. 2 resembles a turtle. Here and in the following we again suppress the channel indices for brevity, and $p$ is the momentum of the incoming baryon. Using the explicit form of $T$ given in eq. (2.33), we find

$$
\begin{aligned}
\Gamma(\not p, \not p) & =\not q \gamma_{5} \hat{g}+T(\not p, \not p-m ; p)\left[(\not p-m) G(p)-I_{\mathrm{M}}\right] \gamma_{5} \hat{g} \\
& =\left[\not \not p p \Gamma_{1}(p)+\not q \Gamma_{2}(p)+\not p \Gamma_{3}(p)+\Gamma_{4}(p)\right] \gamma_{5}
\end{aligned}
$$

with scalar coefficient functions $\Gamma_{n}$ given by

$$
\begin{aligned}
\Gamma_{1}(p)= & T_{1}\left(p^{2} H_{1}-m H_{0}\right)+T_{2}\left(H_{0}-m H_{1}\right) \\
& +T_{4} H_{0}+T_{6} H_{1} \\
\Gamma_{2}(p)= & \hat{g}+p^{2} T_{1}\left(H_{0}-m H_{1}\right)+T_{2}\left(p^{2} H_{1}-m H_{0}\right) \\
& +p^{2} T_{4} H_{1}+T_{6} H_{0} \\
\Gamma_{3}(p)= & T_{3}\left(p^{2} H_{1}-m H_{0}\right)+T_{5}\left(H_{0}-m H_{1}\right) \\
& +T_{7} H_{0}+T_{8} H_{1} \\
\Gamma_{4}(p)= & p^{2} T_{3}\left(H_{0}-m H_{1}\right)+T_{5}\left(p^{2} H_{1}-m H_{0}\right) \\
& +p^{2} T_{7} H_{1}+T_{8} H_{0}
\end{aligned}
$$

where

$$
\begin{aligned}
& H_{1}=\left(G_{0}(p)-m G_{1}(p)\right) \hat{g}, \\
& H_{0}=\left(p^{2} G_{1}(p)-m G_{0}(p)-I_{\mathrm{M}}\right) \hat{g} .
\end{aligned}
$$

The functions $G_{0,1}(p)$ have been defined in eq. (2.23).

In order to complete step (1), we still have to specify which meson-baryon channels contribute in the framework of our electroproduction model. In this first study, we choose to consider only the ground-state octets of mesons and baryons, respectively. Moreover, from the topology of the hadronic part of the Feynman graph in fig. 2 we can conclude that the meson-baryon pairs must have the charge and strangeness quantum numbers of the proton. This limits the number of channels to six:

$$
p \pi^{0}, n \pi^{+}, p \eta, \Lambda K^{+}, \Sigma^{0} K^{+}, \Sigma^{+} K^{0} .
$$

The limitation to these channels can only occur because our amplitude is not crossing-symmetric, otherwise more channels with different quantum numbers must be considered. The violation of crossing-symmetry is a drawback of the BSE method which we use to iterate the rescattering graphs. To our knowledge, the implementation of both crossing symmetry and exact (two-body) unitarity on the basis of Feynman diagrams has not yet been accomplished. Other methods such as, e.g., an analog of the Roy equations (or generalizations thereof) for pion-pion, pion-kaon or pion-nucleon scattering, see [31-33], might be required to achieve this. Here, our goal is more modest, and we sacrifice crossing symmetry in favor of unitarity.

By now, we have finished the first part (step (1)) of our program. Our next task is to couple the photon to the hadronic part of the amplitude in a gauge-invariant fashion. Inserting the photon coupling at every possible place leads to the set of diagrams displayed in fig. 3 .

The first three graphs are generated by coupling the photon to the external lines of the hadronic part. This leads to the expressions

$$
\begin{aligned}
& S_{s}^{\mu}=\Gamma(\not q, \not p) i S(\not p)\left(i e \gamma^{\mu}\right), \\
& S_{u}^{\mu}=\left(i e Q_{\mathrm{B}} \gamma^{\mu}\right) i S\left(\not p_{1}-\not q\right) \Gamma\left(\not d, \not p_{1}\right), \\
& S_{t}^{\mu}=i e Q_{\mathrm{M}}(2 q-k)^{\mu} i \Delta(q-k) \Gamma\left(\not q-\not k, \not p_{1}\right) .
\end{aligned}
$$

Here and in the following, $k$ is the four-momentum of the incoming photon, $q$ is the four-momentum of the outgoing Goldstone boson and $p_{1}$ and $p_{2}$ are the four-momenta of the incoming and outgoing baryon, respectively, while $p \equiv p_{1}+k$. We have also introduced the meson (M) and baryon (B) charge matrices. They are diagonal matrices in channel space and read

$$
\begin{aligned}
& Q_{\mathrm{M}}=\operatorname{diag}(0,1,0,1,1,0), \\
& Q_{\mathrm{B}}=\operatorname{diag}(1,0,1,0,0,1) .
\end{aligned}
$$

The expressions for the three amplitudes $S_{s, u, t}^{\mu}$ must be decomposed into their independent Lorentz structures which is outlined in App. C. Next we consider the coupling of the photon to internal lines of the bubble chain, leading to those diagrams that are most tedious to work out. Consider first the coupling of the photon to an internal baryon line, see fig. 3E. Using the pertinent Feynman rules, the contribution of this graph is easily seen to be

$$
\begin{aligned}
& S_{\mathrm{B}}^{\mu}=-i \int \frac{d^{d} l}{(2 \pi)^{d}} T(\not l, l ; p) S(\not p-\not l) \Delta(l) e Q_{\mathrm{B}} \gamma^{\mu} \\
& \times S\left(\not p_{1}-\not l\right) \Gamma\left(\not l, \not p_{1}\right),
\end{aligned}
$$

while the coupling of the photon to an internal meson line (fig. 3D) leads to

$$
\begin{aligned}
S_{\mathrm{M}}^{\mu}= & -i \int \frac{d^{d} l}{(2 \pi)^{d}} T(\not p, \not p-\not p ; p) S(\not) \Delta(p-l) e Q_{\mathrm{M}} \\
& \times\left(2\left(p_{1}-l\right)+k\right)^{\mu} \Delta\left(p_{1}-l\right) \Gamma\left(\not p_{1}-\not l, \not p_{1}\right) .
\end{aligned}
$$

Again, the decomposition into independent Lorentz structures can be found in App. C 


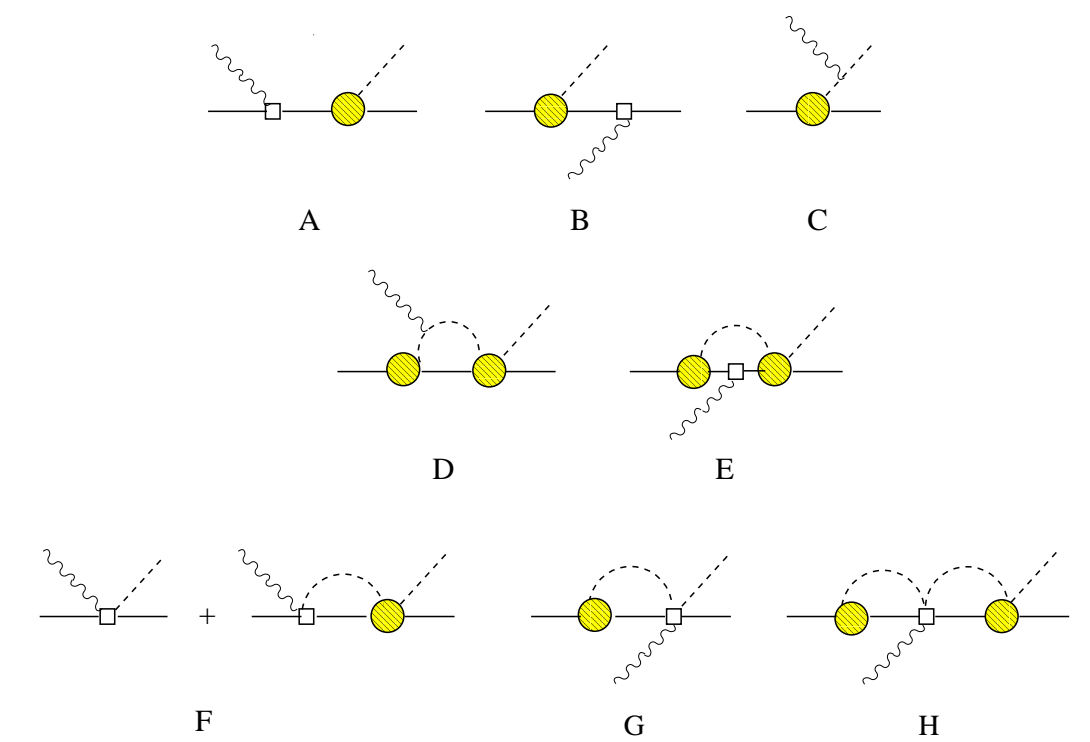

Fig. 3. Classes of diagrams for kaon production off the nucleon in the turtle approximation utilized here.

The next class of graphs we consider arises due to the 'Kroll-Ruderman' (KR) term stemming from the covariant derivative $\nabla_{\mu} U$ in the chiral Lagrangian. The corresponding tree level vertex reads (in matrix form)

$$
S_{\mathrm{KR}, \mathrm{tree}}^{\mu}=e Q_{\mathrm{M}} \hat{g} \gamma^{\mu} \gamma_{5}
$$

We add the loop contribution that accounts for the finalstate interaction after the meson has left the KR vertex, to arrive at (see fig. 3. F)

$S_{\mathrm{KR}}^{\mu}=S_{\mathrm{KR}, \text { tree }}^{\mu}+\int \frac{d^{d} l}{(2 \pi)^{d}} T(\not l, l ; p) i S(\not p-\not) \Delta(l) S_{\mathrm{KR}, \text { tree }}^{\mu}$.

The remaining graphs are depicted in figs. 3, G,H. These contributions arise from the terms proportional to the external vector field in the chiral connection $\Gamma^{\mu}$, leading to a $\bar{B} \phi \phi \gamma B$ vertex rule

$$
-i e \gamma^{\mu}\left\{Q_{\mathrm{M}}, g\right\}
$$

and to the following expressions for the Feynman graphs of fig. [3 G,H:

$$
\begin{aligned}
& S_{\mathrm{WT} 1}^{\mu}=e \gamma^{\mu}\left\{Q_{\mathrm{M}}, g\right\} \int \frac{d^{d} l}{(2 \pi)^{d}} i S\left(\not \not_{1}-\not l\right) \Delta(l) \Gamma\left(l, \not p_{1}\right), \\
& S_{\mathrm{WT} 2}^{\mu}=\int \frac{d^{d} \tilde{l}}{(2 \pi)^{d}} T(\not \phi, \tilde{l} ; p) i S(\not p-\tilde{l}) \Delta(\tilde{l}) S_{\mathrm{WT} 1}^{\mu}
\end{aligned}
$$

The Lorentz structure decomposition of all the above diagrams and the according contributions to the invariant amplitudes $B_{i}$ are given in App. C.

Having finished the construction of the electroproduction amplitude, we return to the issue of unitarity. The crucial observation here is that every electroproduction amplitude $\mathcal{M}^{\mu}(q, k ; p)$ which may be written as (see also

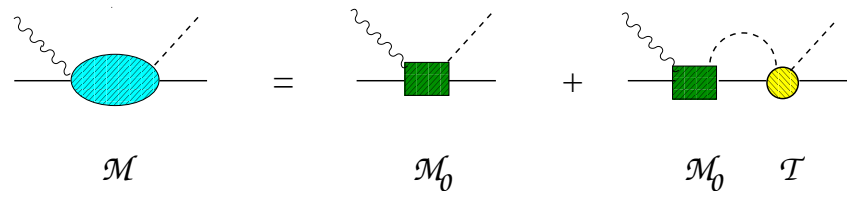

Fig. 4. Illustration of the integral equation (3.20) satisfied by our electroproduction amplitude

fig. (4)

$$
\begin{aligned}
\mathcal{M}^{\mu}(q, k ; p) & =\mathcal{M}_{0}^{\mu}(q, k ; p) \\
& +\int \frac{d^{d} l}{(2 \pi)^{d}} \mathcal{T}(q, l ; p) i S(\not p-\not) \Delta(l) \mathcal{M}_{0}^{\mu}(l, k ; p)
\end{aligned}
$$

obeys the requirement of two-body unitarity in the subspace of meson-baryon channels. Here, $\mathcal{T}$ is an amplitude for meson-baryon scattering that solves a BSE of the type of eq. (2.9) and $\mathcal{M}_{0}^{\mu}$ is a real kernel.

The proof proceeds in close analogy to the one presented in sect. 5 of [25]. Now we note that our electroproduction amplitude given above decomposes into five 'unitarity classes', each of which obey an integral equation of the above type (3.20) by construction (with $\mathcal{T}=T$ ):

$$
\begin{aligned}
& \text { Class } 1: S_{s}^{\mu} \\
& \text { Class } 2: S_{u}^{\mu}+S_{\mathrm{B}}^{\mu} \\
& \text { Class } 3: S_{t}^{\mu}+S_{\mathrm{M}}^{\mu} \\
& \text { Class } 4: S_{\mathrm{KR}}^{\mu} \\
& \text { Class } 5: S_{\mathrm{WT} 1}^{\mu}+S_{\mathrm{WT} 2}^{\mu} .
\end{aligned}
$$

Hence, each class leads to a electroproduction amplitude that obeys unitarity for itself, though not gauge invariance. This is because the expressions $S_{\mathrm{WT} 1}^{\mu}, S_{t}^{\mu}$ and $S_{u}^{\mu}$ as well as the tree graphs of $S_{\mathrm{KR}}^{\mu}$ and $S_{s}^{\mu}$ are real in the physical region for the electroproduction process and can 
therefore constitute a kernel $\mathcal{M}_{0}^{\mu}$ in eq. (3.20). The sum

$$
\mathcal{M}^{\mu} \equiv S_{s}^{\mu}+S_{u}^{\mu}+S_{t}^{\mu}+S_{\mathrm{B}}^{\mu}+S_{\mathrm{M}}^{\mu}+S_{\mathrm{KR}}^{\mu}+S_{\mathrm{WT} 1}^{\mu}+S_{\mathrm{WT} 2}^{\mu}
$$

will then also obey unitarity, due to the linearity of the integral equation (3.20) in $\mathcal{M}_{0}^{\mu}$.

We will now show that the sum $\mathcal{M}^{\mu}$ of the five classes is gauge invariant by proving $k \cdot \mathcal{M}=0$ for on-shell mesons and baryons. This might be obvious from our construction, but we include the proof for completeness. The contraction of $k$ with the different amplitudes yields

$$
\begin{aligned}
& k \cdot S_{s}=-e \Gamma(\not q, \not p), \\
& k \cdot S_{u}=e Q_{\mathrm{B}} \Gamma\left(\not, \not \phi_{1}\right) \text {, } \\
& k \cdot S_{t}=e Q_{\mathrm{M}} \Gamma\left(\not q-\not k, \not p_{1}\right), \\
& k \cdot S_{\mathrm{B}}=\int \frac{d^{d} l}{(2 \pi)^{d}} T(\not q, l ; p) i S(\not p-\not l) \Delta(l) e Q_{\mathrm{B}} \Gamma\left(\not l, \not p_{1}\right) \\
& -\int \frac{d^{d} l}{(2 \pi)^{d}} T(\not q, \not ; p) e Q_{\mathrm{B}} i S\left(\not \not_{1}-\not\right) \Delta(l) \Gamma\left(\not l, \not p_{1}\right), \\
& k \cdot S_{\mathrm{M}}=\int \frac{d^{d} l}{(2 \pi)^{d}} T(\not q, l ; p) i S(\not p-\not l) \Delta(l) e Q_{\mathrm{M}} \\
& \times \Gamma\left(l-\not k, \not p_{1}\right) \\
& -\int \frac{d^{d} l}{(2 \pi)^{d}} T(\not l, l+\not k ; p) e Q_{\mathrm{M}} i S\left(\not p_{1}-\not l\right) \\
& \times \Delta(l) \Gamma\left(l, \not p_{1}\right), \\
& k \cdot S_{\mathrm{KR}}=e Q_{\mathrm{M}} \hat{g} \not k \gamma_{5} \\
& +\int \frac{d^{d} l}{(2 \pi)^{d}} T(\not d, l ; p) i S(\not p-\not) \Delta(l) e Q_{\mathrm{M}} \hat{g} k \gamma_{5}, \\
& k \cdot S_{\mathrm{WT} 1}=e \not k\left\{Q_{\mathrm{M}}, g\right\} \int \frac{d^{d} l}{(2 \pi)^{d}} i S\left(\not p_{1}-\not\right) \Delta(l) \Gamma\left(\not l, \not p_{1}\right), \\
& k \cdot S_{\mathrm{WT} 2}=\int \frac{d^{d} \tilde{l}}{(2 \pi)^{d}} T(\not q, \tilde{l} ; p) i S(\not p-\tilde{l}) \Delta(\tilde{l}) e \not k\left\{Q_{\mathrm{M}}, g\right\} \\
& \times \int \frac{d^{d} l}{(2 \pi)^{d}} i S\left(\not p_{1}-\not l\right) \Delta(l) \Gamma\left(l, \not p_{1}\right) .
\end{aligned}
$$

Here, charged external particles are put on-shell. First, we note that the tree graphs are gauge invariant for themselves, since the tree part of $k \cdot\left(S_{s}+S_{u}+S_{t}\right)$ equals

$$
-e \hat{g} \phi \gamma_{5}+e Q_{\mathrm{B}} \hat{g} \phi \gamma_{5}+e Q_{\mathrm{M}} \hat{g}(\not q-\not k) \gamma_{5},
$$

which is exactly canceled by the first term of $k \cdot S_{\mathrm{KR}}$ (recall $\left.Q_{\mathrm{B}}+Q_{\mathrm{M}}=1\right)$. In order to deal with the loop contributions, it is useful to rewrite the integral equations (2.9) and (3.3) for $T$ and $\Gamma$, respectively, as

$$
\begin{aligned}
T(\not d, l ; p) & =g(\not d+\not l) \\
& +\int \frac{d^{d} \tilde{l}}{(2 \pi)^{d}} T(\not q, \not l ; p) i S(\not p-\tilde{l}) \Delta(\tilde{l}) g(\tilde{l}+\not),
\end{aligned}
$$

and

$\Gamma\left(\tilde{l}, \not p_{1}\right)=\hat{g} \tilde{l} \gamma_{5}+\int \frac{d^{d} l}{(2 \pi)^{d}} g(\tilde{l}+\not) i S\left(\not p_{1}-\not l\right) \Delta(l) \Gamma\left(\not l, \not p_{1}\right)$.
These equations are equivalent to eqs. (2.9) and (3.3). Using eq. (3.22) in the second term of $k \cdot S_{\mathrm{B}}$ and $k \cdot S_{\mathrm{M}}$, and eq. (3.23) in the first term of $k \cdot S_{\mathrm{B}}$ and $k \cdot S_{\mathrm{M}}$, it is straightforward to show that the loop contributions in $k \cdot \mathcal{M}$ cancel. This, together with the above result for the tree contributions, completes the proof of gauge invariance for the full amplitude $\mathcal{M}$.

\section{Results}

In this section, we will perform an overall $\chi^{2}$ fit to available photoproduction and pion-induced data on the proton near the respective thresholds. In more detail, we fit the differential cross sections for photoproduction on the proton into the $K^{+} \Lambda, K^{+} \Sigma^{0}, K^{0} \Sigma^{+}$final states as well as of $\pi^{-} p \rightarrow K^{0} \Lambda, K^{0} \Sigma^{0}$. Inspection of the differential cross sections reveals that already at moderate energies away from threshold $p$ - and $d$-waves become increasingly important. Since our approach, which is based on the WeinbergTomozawa interaction kernel, generates mainly $s$-waves (with one important exception discussed below) and thus does not provide a realistic description for higher partial waves, we expect it to be valid only in the near-threshold regions. We have thus restricted our fits to energy values for which the differential cross sections are dominated by $s$-waves, i.e. center-of-mass energies of about $1.80 \mathrm{GeV}$ corresponding to photon lab momenta of about $1.25 \mathrm{GeV}$ or pion lab momenta of about $1.23 \mathrm{GeV}$. Still, we will be able to extract interesting information from such investigations, in particular, we can study in detail the commonly appearing approximations made in the literature as already mentioned in the introduction. The extension to higher energies requires inclusion of higher order counter terms from the effective Lagrangian in the derivation of the interaction kernel. However, this is beyond the scope of the present work. In this investigation, we focus on the importance of gauge invariance and study the on-shell approximation in the driving terms and are satisfied with a moderate description of both photoproduction and pioninduced data on the proton near threshold. Our investigation sets the stage for systematic improvements in the future which will lead to better agreement with experiment.

The free parameters in our approach are, on the one hand, the three meson decay constants $F_{\pi}, F_{K}, F_{\eta}$ which we vary separately within realistic bounds, as the $\mathrm{SU}(3)$ symmetry-breaking differences between them are beyond our working precision of the effective potential. More precisely, $\mathrm{SU}(3)$ symmetry breaking is generated by various higher order terms in the meson-baryon (meson) Lagrangian starting at chiral order two (four). Since these contributions are not included in the leading order WT kernel, we simulate such effects by allowing variations in the various meson decays constants. This, of course, will no longer be done when the higher order terms in the interaction kernel have been included. One observes that the fitted decay constants tend to larger values reducing the strength of the Weinberg-Tomozawa interaction. This is consistent with findings in earlier chiral unitary studies, 

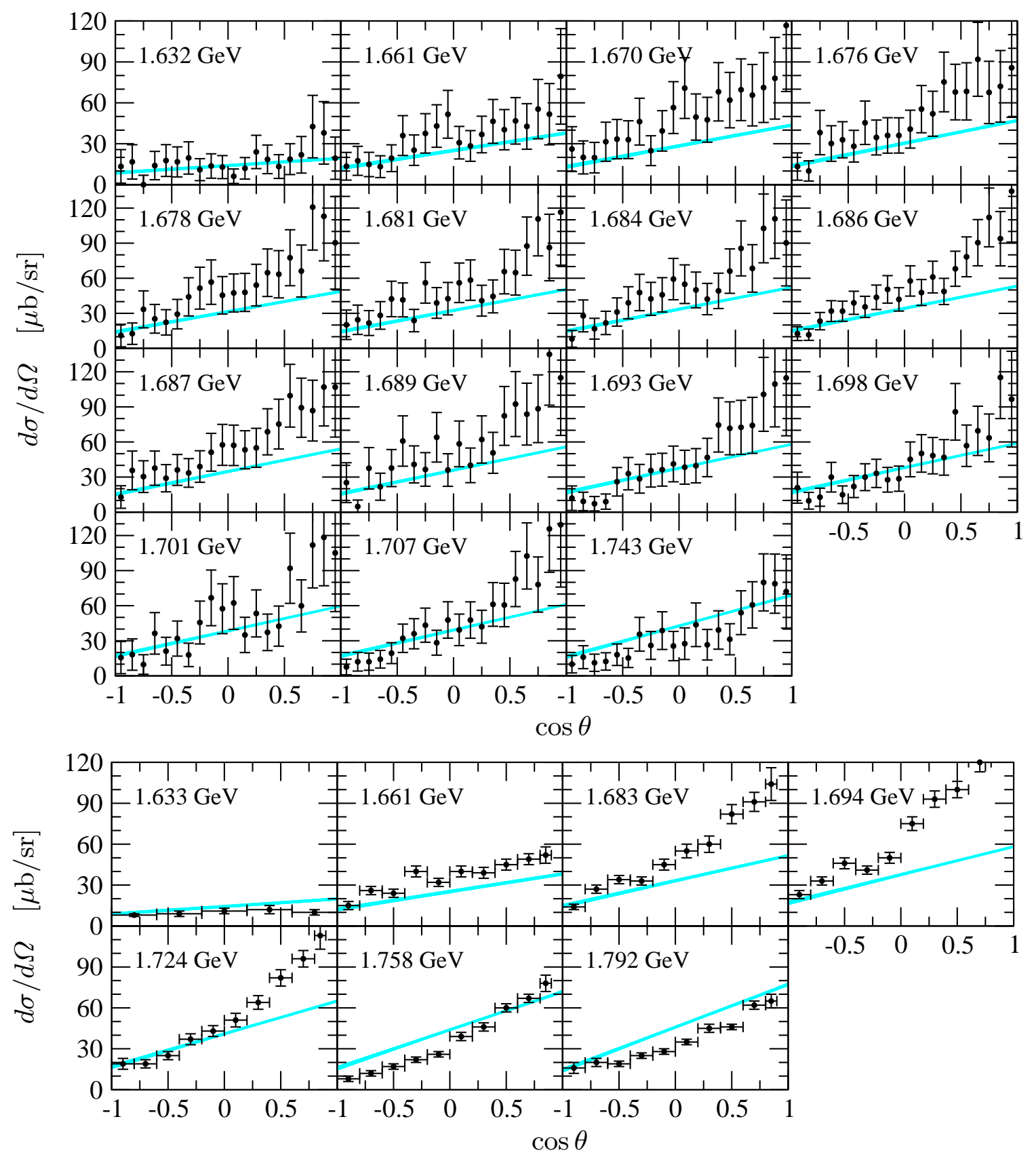

Fig. 5. Differential cross sections for $\pi^{-} p \rightarrow K^{0} \Lambda$ compared to data from [34] (upper panel) and [35] (lower panel). The number in each plot denotes the respective c.m. energy $\sqrt{s}$.

i.e. the WT interaction in many cases produces too strong $s$-waves, see e.g. [24]. We allow $F_{\pi}, F_{K}$, and $F_{\eta}$ to vary between $70 \mathrm{MeV}$ and $150 \mathrm{MeV}$. The values we find for the best fits, i.e. the fits with the lowest overall $\chi^{2}$ values, are

$$
\begin{aligned}
F_{\pi} & =(113 \ldots 127) \mathrm{MeV}, \\
F_{K} & =(149 \ldots 150) \mathrm{MeV}, \\
F_{\eta} & =(74 \ldots 82) \mathrm{MeV} .
\end{aligned}
$$

Note that $F_{K}$ tends towards the upper limit of $150 \mathrm{MeV}$. On the other hand, we fit the four different isospin-symmetric scales $\mu$ in the loop integrals. For the best fits their values are:

$$
\begin{aligned}
\mu_{\pi N} & =(0.46 \ldots 0.54) \mathrm{GeV}, \\
\mu_{\eta N} & =(3.29 \ldots 4.41) \mathrm{GeV}, \\
\mu_{K \Lambda} & =(2.56 \ldots 2.86) \mathrm{GeV}, \\
\mu_{K \Sigma} & =(3.66 \ldots 4.31) \mathrm{GeV} .
\end{aligned}
$$

We note that these values are roughly in accordance with the natural size estimate of ref. [36] (although most of them turn out to be somewhat large).

In figs. 5, 6] we present the best fit results for the pioninduced differential cross sections $\pi^{-} p \rightarrow K^{0} \Lambda, K^{0} \Sigma^{0}$, 


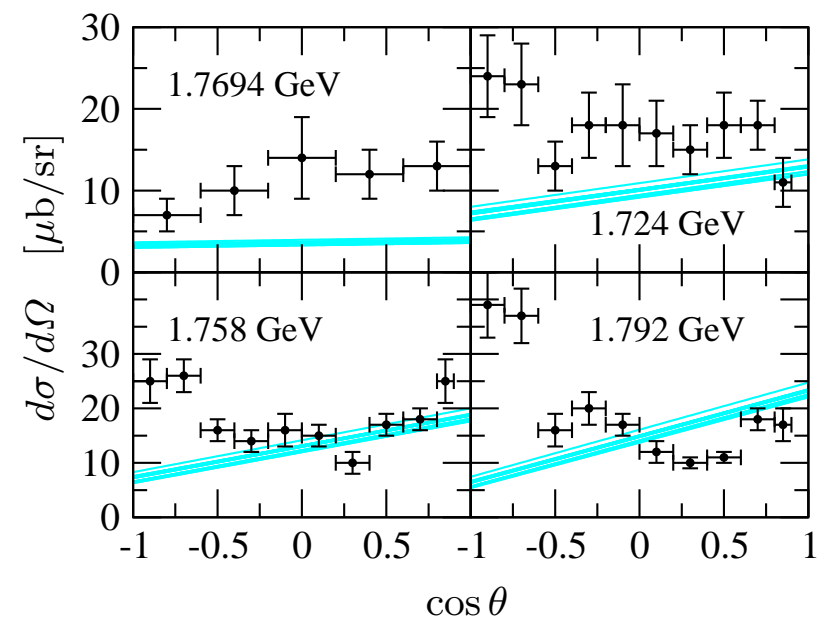

Fig. 6. Differential cross sections for $\pi^{-} p \rightarrow K^{0} \Sigma^{0}$ compared to data from [38]. The number in each plot denotes the respective c.m. energy $\sqrt{s}$.

respectively. Although the WT interaction kernel is entirely $s$-wave, there are $p$-wave contributions due to the lower components of the Dirac spinors which are introduced in the calculation of the scattering matrix $T$. The corresponding total cross sections and the one for $\pi^{-} p \rightarrow$ $K^{+} \Sigma^{-}$are shown in fig. 7. We remark that the bands in these figures are generated by about 20 fits with a $\chi^{2}$ very close to its minimal value. Since with the simple WT interaction we are not able to describe all these and the photon-induced data to a high accuracy, we refrain from giving one-sigma error bands as done in our study on $K^{-} p$ scattering [37]. This will be done in future work when the higher order terms in the interaction kernel will be included.

The processes $\pi^{-} p \rightarrow K^{+} \Sigma^{-}$and $\pi^{+} p \rightarrow K^{+} \Sigma^{+}$are dominated by $p$ - and $d$-waves already close to threshold. Hence, a realistic description of these channels cannot be provided by the leading WT interaction. In order not to overestimate the data we have still included the total cross section for $\pi^{-} p \rightarrow K^{+} \Sigma^{-}$in our fit. We refrain, however, from taking into account data on $\pi^{+} p \rightarrow K^{+} \Sigma^{+}$at all since, regardless of the choice of parameters (within realistic ranges), our approach clearly overshoots the $\pi^{+} p \rightarrow$ $K^{+} \Sigma^{+}$cross section. This indicates that higher order contact interactions in the interaction kernel are absolutely necessary - particularly for this process - in order to reduce the strength of the WT term. Moreover, it is worthwhile mentioning that the presented fits exhibit a peak in the $\pi^{-} p \rightarrow \eta n$ cross section due to the $S_{11}(1535)$ resonance. Pion- and photon-induced eta production data will be included in future work after the interaction kernel has been developed to higher chiral orders.

Furthermore, we have included in our fit data on differential cross sections of the photoproduction processes $\gamma p \rightarrow K^{+} \Lambda, K^{+} \Sigma^{0}, K^{0} \Sigma^{+}$. The results are displayed in figs. 8, 9, 10, respectively, while the corresponding total cross sections are presented in fig. 11. A few remarks are in order: The SAPHIR and CLAS data on charged kaon photoproduction show some inconsistencies at forward angles,

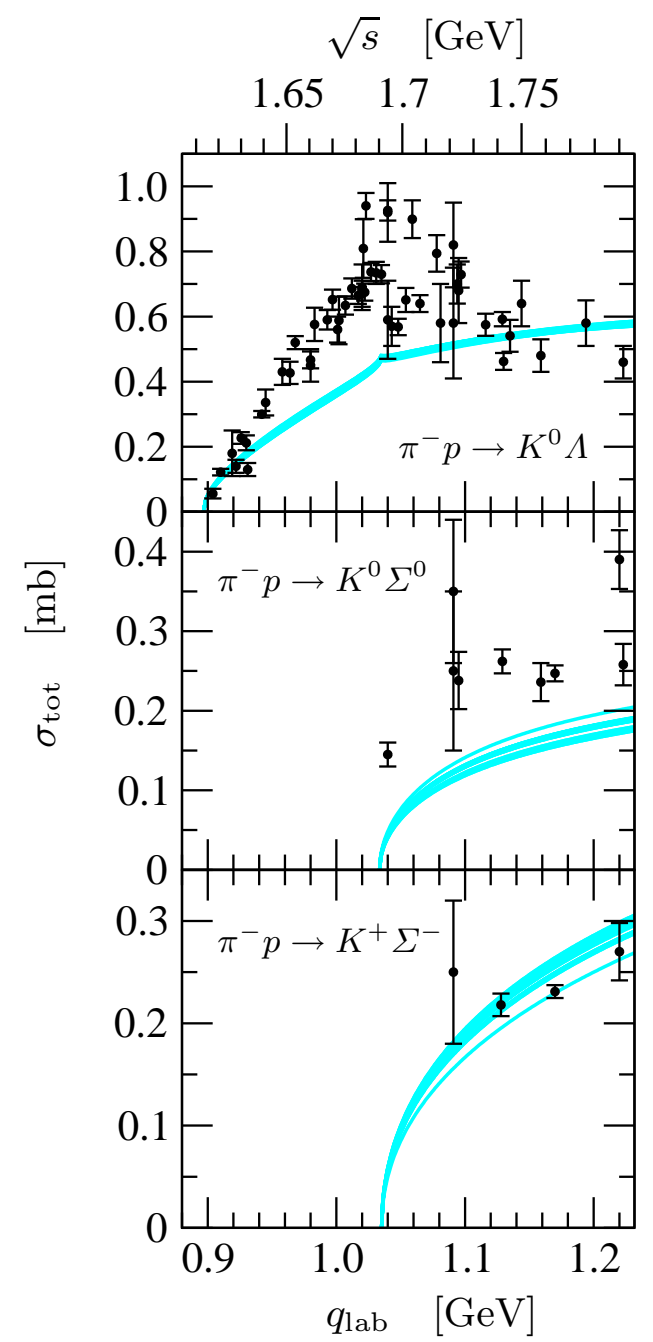

Fig. 7. Total cross sections for $\pi^{-}$induced production of $K^{0} \Lambda$ (top), $K^{0} \Sigma^{0}$ (middle), $K^{+} \Sigma^{-}$(bottom) as a function of the pion lab momentum $q_{\text {lab }}$. The corresponding c.m. energy $\sqrt{s}$ is also given. The data are taken from $[34,35,38]$ and the compilation [39].

but this can not be resolved within the approximations made here. Also, the very different shape of the differential cross sections for the $K^{+} \Lambda$ and $K^{+} \Sigma^{0}$ final states can be traced back to the isospin selectivity of the $\Lambda$, see also ref. [40].

To summarize, the results presented here show a reasonable agreement with data on photon- and pion-induced reactions close to threshold, but more realistic interaction kernels and higher partial waves are required to obtain better agreement with data, in particular away from threshold. This is however beyond the scope of the present investigation.

Of interest here are gauge invariance violations encountered in previous chiral unitary approaches which only took a subset of the diagrams in fig. [3into account. In order to estimate the typical size of gauge symmetry violations, we omit all contributions where the photon couples to intermediate and final states and retain only those dia- 

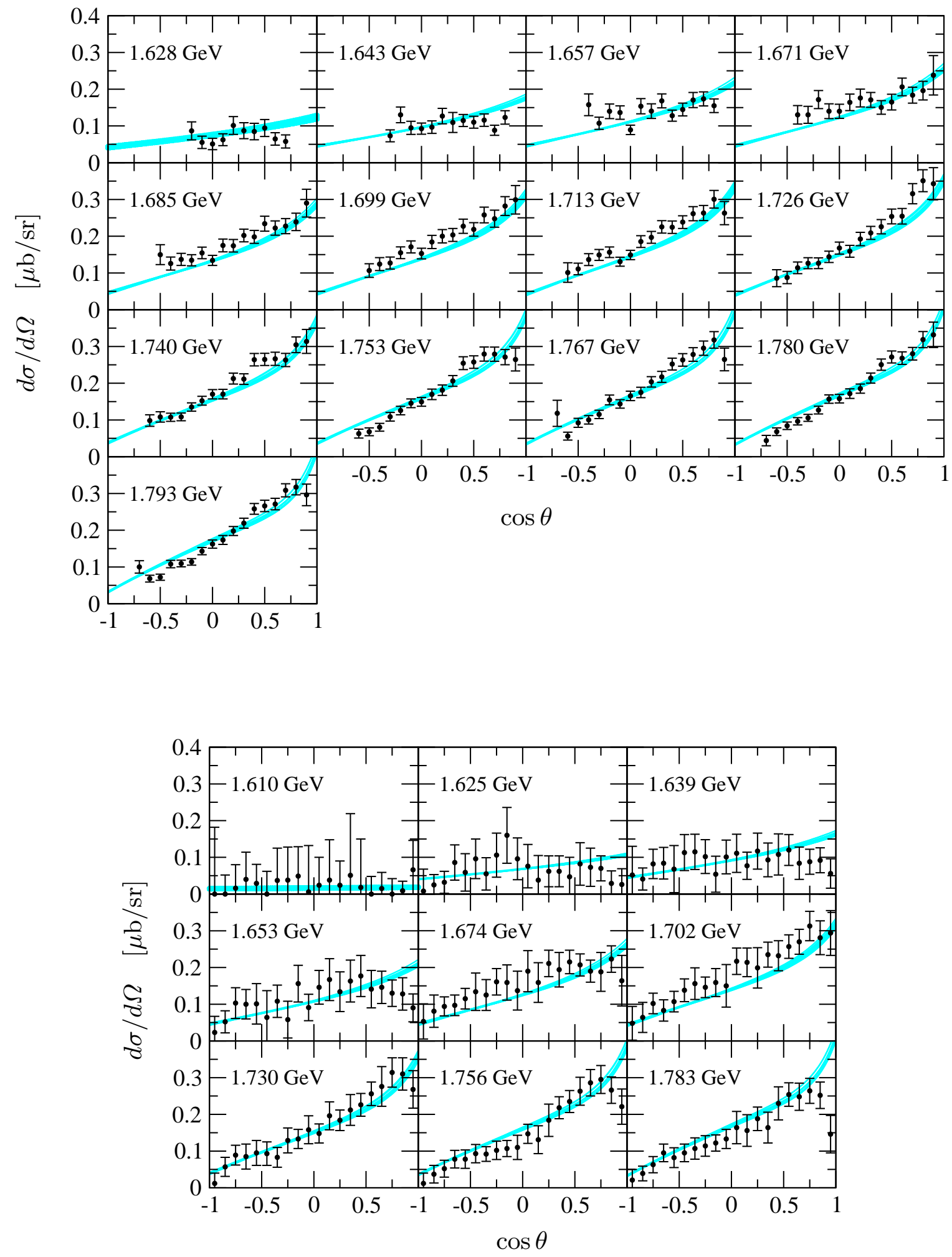

Fig. 8. Differential cross sections for $\gamma p \rightarrow K^{+} \Lambda$ compared to data from [7] (upper panel) and [4] (lower panel). The number in each plot denotes the respective c.m. energy $\sqrt{s}$. 

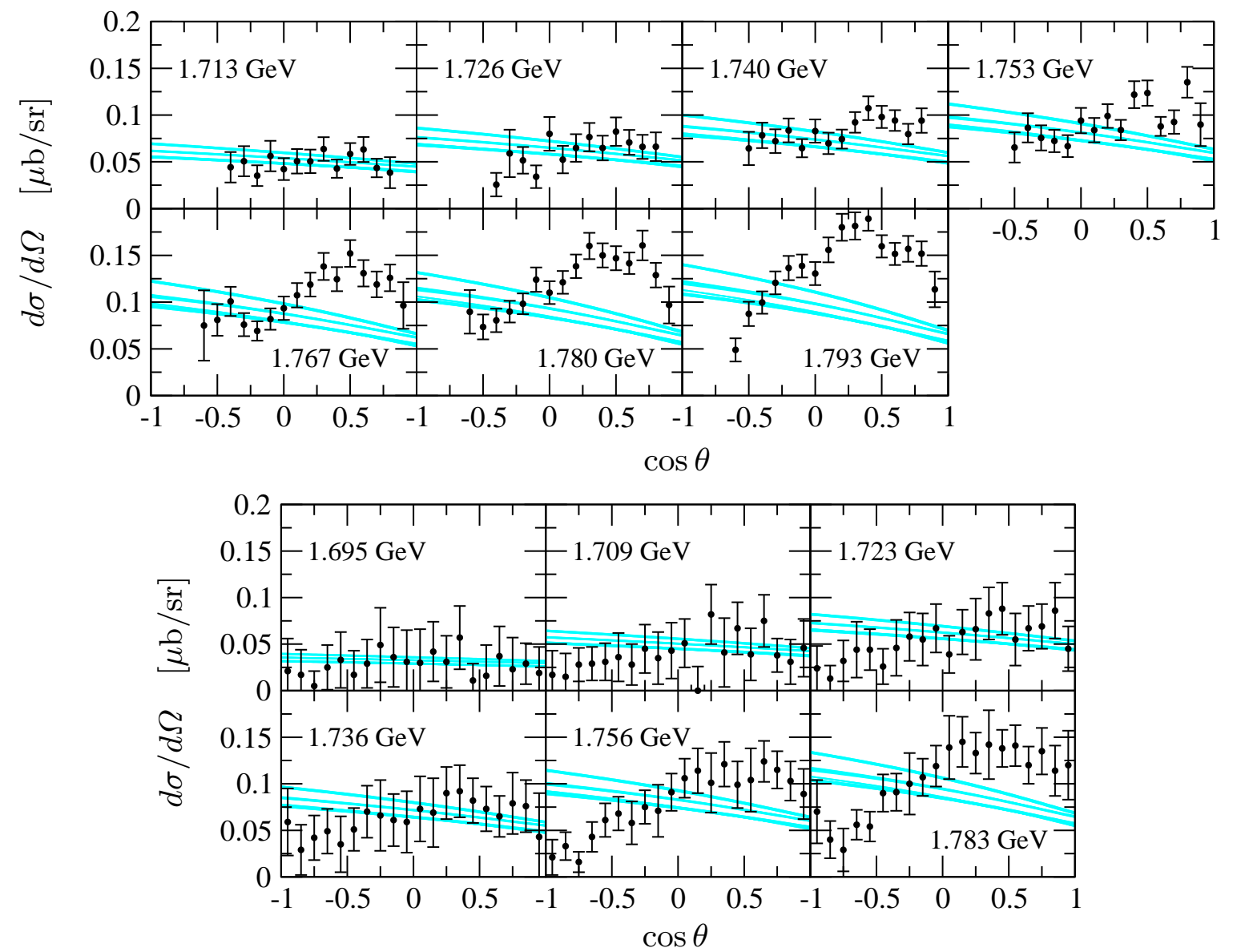

Fig. 9. Differential cross sections for $\gamma p \rightarrow K^{+} \Sigma^{0}$ compared to data from [7] (upper panel) and [4] (lower panel). The number in each plot denotes the respective c.m. energy $\sqrt{s}$.

grams where the photon is initially absorbed (figs. $3 \mathrm{~A}+\mathrm{F}$ ). Note that both the graphs A and F as well as their sum are unitary as explained in detail in sect. 3. For making this comparison, we do not refit the parameters but use the values obtained in the full approach. Some sample results are compared to the full approach in fig. 12. One clearly observes that violations of gauge invariance are sizable, although this effect could, in principle, be concealed numerically by readjusting the parameters of the approach. This indicates that the additional contributions which render the photoproduction amplitudes gauge invariant and were omitted in previous work are not negligible and must be taken into account.

In order to be able to compare our results with previous chiral unitary approaches we have also worked in the approximations employed in these investigations, see e.g. [24]1. To this aim, the interaction kernel for mesonbaryon scattering is directly sandwiched between Dirac spinors and projected onto the $s$-wave which is then iterated to infinite order in a geometric series. Furthermore, for the photoproduction process only the leading $s$-wave

\footnotetext{
1 Note, however, that in ref. [24] the primary goal was the consistent inclusion of the $\eta^{\prime}$ meson in chiral unitary approaches.
}

(the so-called $E_{0+}$ multipole) is considered. Obviously, this scheme produces pure $s$-waves and cannot reproduce the structures from higher partial waves in the differential cross sections, see fig. 13. One notes that these approximations can indeed be sizable. Of course, in ref. [24] higher order terms were included in the $E_{0+}$ multipole, but that does not change the observations just made. Again, by a suitable parameter refitting one might be able to describe the total cross sections, but given the more sophisticated scheme developed here, such approximations are no longer necessary.

Overall, we have illustrated that both the on-shell approximation and the omission of certain classes of diagrams which are required to fulfill gauge invariance constitute crude approximations utilized within chiral unitary approaches in the past. Although these effects can numerically be concealed to a large extent by readjusting the parameters, important theoretical constraints are not met in this manner. On the other hand, the approach we have developed here is in accordance with these criteria. We stress again that our results also show that it is mandatory to go beyond the leading WT approximation in the interaction kernel and to properly include higher partial waves in the BSE. Moreover, contributions from three-particle intermediate states, such as $\pi \pi N$, might play a role in kaon 


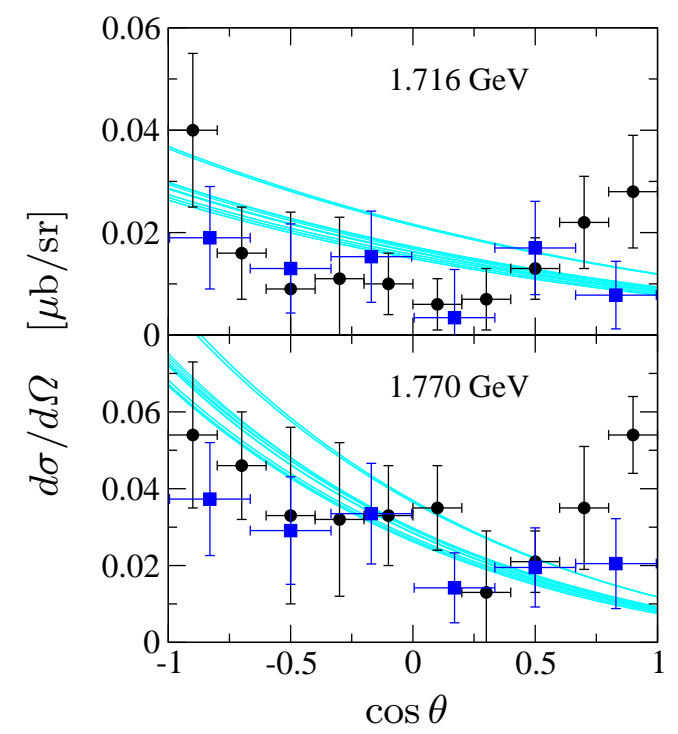

Fig. 10. Differential cross sections for $\gamma p \rightarrow K^{0} \Sigma^{+}$compared to data from [5] (circles) and [8] (squares). The number in each plot denotes the respective c.m. energy $\sqrt{s}$.

photoproduction, see e.g. [41], but are beyond the scope of the present investigation and have thus been discarded. For a method to incorporate such three-particle cuts, see ref. [42].

\section{Conclusions and outlook}

The search for missing resonances is of great importance and actively being pursued at various experimental facilities. A promising tool to discover new resonances is the photoproduction of kaons on protons. A strong coupling to channels with open strangeness could reveal new resonances yet undetected in previous experiments based on pion-nucleon physics. Experiments with open strangeness are currently performed at ELSA, JLab and at SPring8 providing a host of experimental data. The obtained data must be met by theoretical approaches and yield a set of tight constraints. In this work, a chiral unitary approach based on the combination of the chiral effective Lagrangian with a coupled-channels Bethe-Salpeter equation is presented. The method is exactly unitary and satisfies gauge invariance. It improves previous approaches in this field which employed only a subset of the Feynman diagrams considered here.

We have fitted both the differential cross sections for photoproduction into $K^{+} \Lambda, K^{+} \Sigma^{0}, K^{0} \Sigma^{+}$as well as of the meson-baryon scattering processes $\pi^{-} p \rightarrow K^{0} \Lambda, K^{0} \Sigma^{0}$ $K^{+} \Sigma^{-}$. In the fits, we have restricted ourselves to the threshold regions of the respective channels, as we cannot expect to obtain a realistic description of these processes away from threshold due to the increasing importance of higher partial waves absent in the Weinberg-Tomozawa interaction kernel. In order to improve the agreement with experimental data, the inclusion of higher chiral orders in the interaction kernel is necessary. In charged kaon photo-

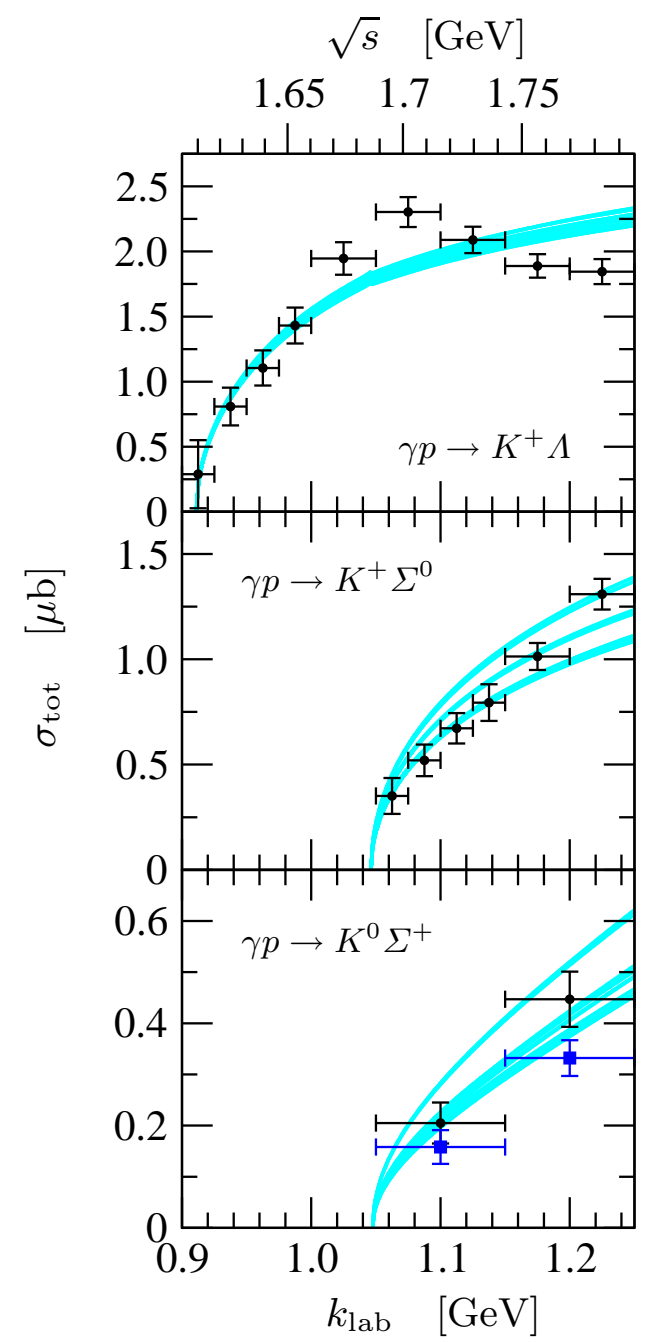

Fig. 11. Total cross sections for photoproduction of $K^{+} \Lambda$ (top), $K^{+} \Sigma^{0}$ (middle), $K^{+} \Sigma^{0}$ (bottom) as a function of the

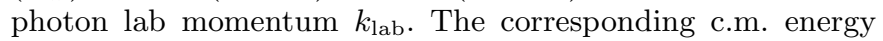
$\sqrt{s}$ is also given. The data are taken from [5] (circles) and [8] (squares).

production, important contributions to the $p$ and higher partial waves are generated by the kaon pole term, which is already included at the order we are working.

Our aim was to estimate, on the one hand, the size of gauge invariance violations introduced in previous coupled-channels analyses by neglecting Feynman diagrams where the photon couples to intermediate states of the Bethe-Salpeter bubble chain. On the other hand, the simplification of setting the interaction kernel on-shell and performing $s$-wave projections on the meson-baryon and the photon-baryon subsystems is studied. Our investigation suggests that both approximations are not justified in the treatment of photoproduction processes and lead to sizable changes in the results. It is thus important to satisfy gauge invariance and include off-shell terms in the effective potential. The approach presented here fulfills these requirements in a minimal way, i.e. any subset of 

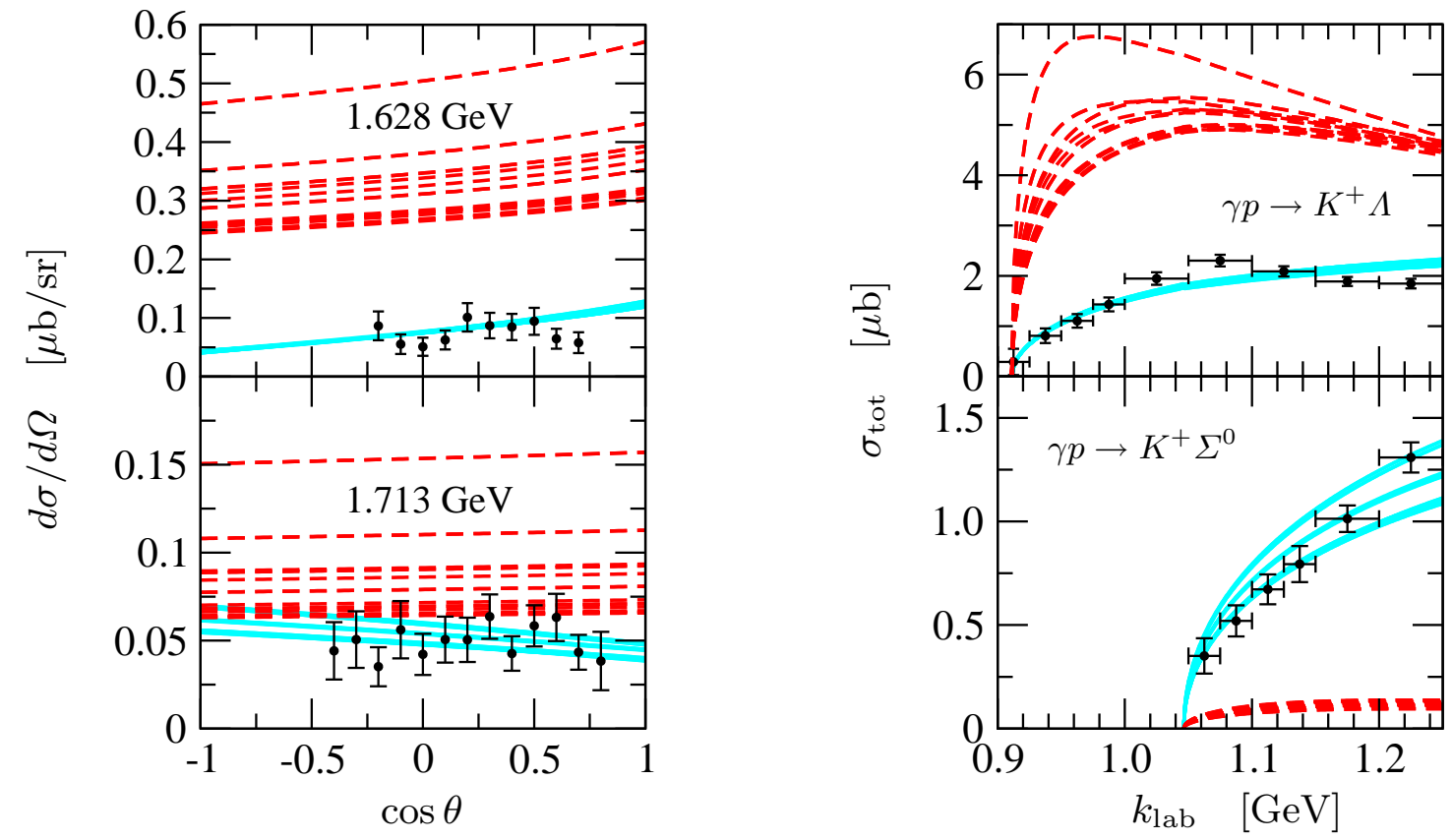

Fig. 12. Sample results which illustrate the importance of contributions which are needed to fulfill gauge invariance. Left panel: differential cross sections for photoproduction of $K^{+} \Lambda$ (top), $K^{+} \Sigma^{0}$ (bottom) at c.m. energies stated in the figures compared to data from [7]. Right panel: total cross sections for photoproduction of $K^{+} \Lambda$ (top), $K^{+} \Sigma^{0}$ (bottom) compared to data from [4]. The full result is represented by the solid lines, whereas the dashed lines indicate the contributions from diagrams $\mathrm{A}+\mathrm{F}$ in fig. 3
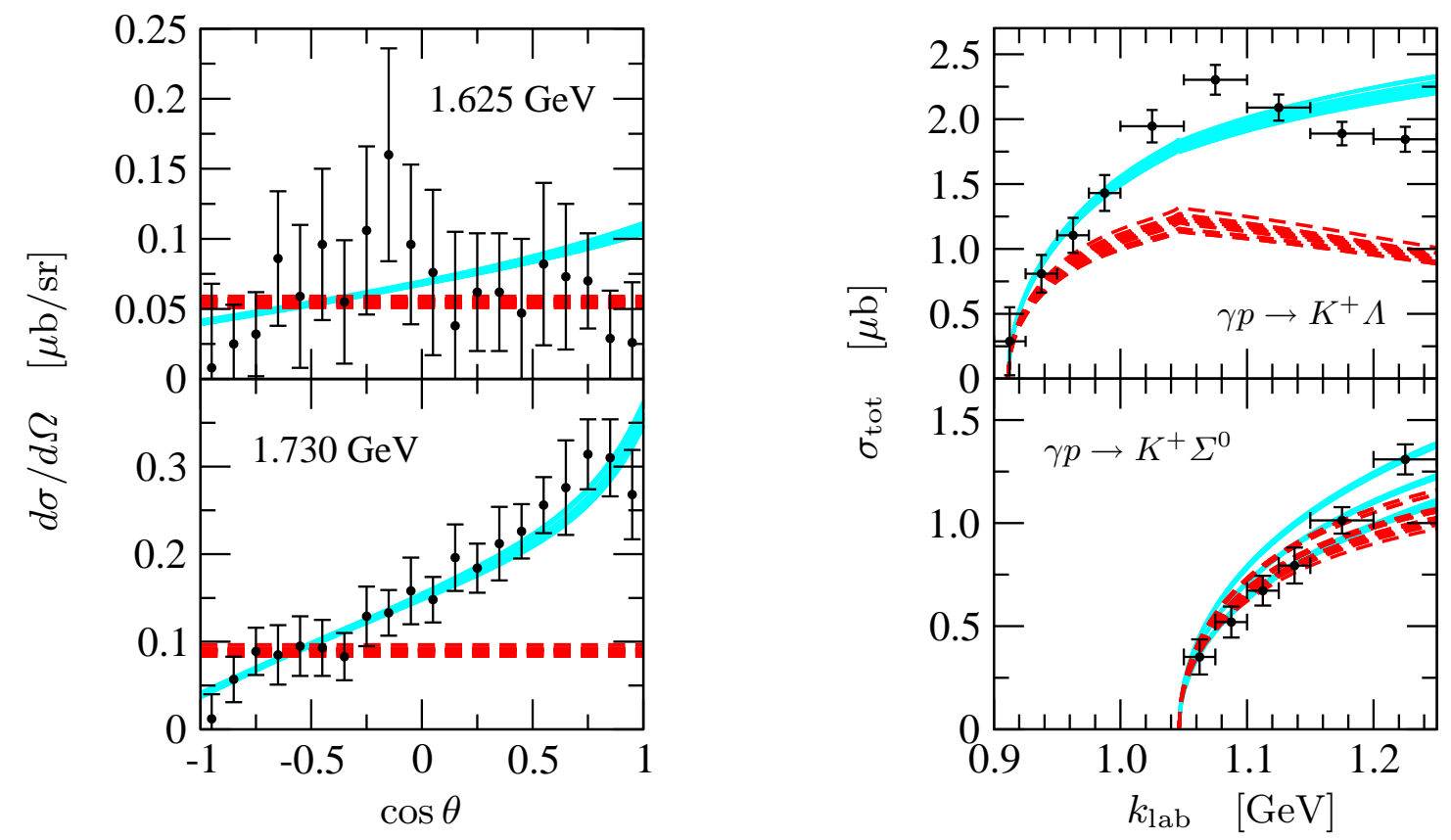

Fig. 13. Sample results which illustrate the importance of contributions neglected in conventional coupled-channels analyses. Left panel: differential cross sections for photoproduction of $K^{+} \Lambda$ at two different c.m. energies as stated in the figures. Right panel: total cross sections for photoproduction of $K^{+} \Lambda$ (top), $K^{+} \Sigma^{0}$ (bottom). The full result is represented by the solid lines, whereas the dashed lines correspond to the outcome of the model [24] given the same values of the fit parameters. The data are taken from [4]. 
the included diagrams cannot be omitted as this would violate either exact unitarity or gauge invariance.

There are three directions in which the approach presented here needs to be improved: First, the inclusion of higher order terms in the interaction kernel and the resulting better inclusion of $p$-waves and higher multipoles is straightforward but tedious. This will allow to include also the polarization data into the analysis. In this context, we should also mention that in the chiral unitary approach not all resonances are generated dynamically, so in fact one might have to include explicit resonance fields in certain channels to achieve a precise description. Such a method has already been developed for elastic pionnucleon scattering, see ref. [36]. Second, one should also include pion and photon induced $\eta, \eta^{\prime}$ production data as already accomplished in [24] and further constrain the scattering and production amplitudes through matching to the two-flavor sector (as done $e . g$. in refs. [43,44]). Last but not least, the violation of crossing symmetry due to the turtle approximation needs to be repaired. This could, in principle, be done by formulating Roy-type equations, but is technically involved.

\section{Acknowledgements}

We thank Ulrike Thoma for useful discussions and for providing us with the CB-ELSA/TAPS data. This work is supported in part by DFG (SFB/TR 16, "Subnuclear Structure of Matter", and BO 1481/6-1) and is part of the EU Integrated Infrastructure Initiative Hadron Physics Project under contract number RII3-CT-2004-506078.

\section{A Bethe-Salpeter approach with off-shell kernel}

In this appendix, we present an alternative solution of the Bethe-Salpeter equation with off-shell pieces in the interaction kernel $[45,46]$. In general, the amplitude for a meson-baryon scattering process $B_{a}\left(p_{a}\right)+\phi_{i}\left(q_{i}\right) \rightarrow B_{b}\left(p_{b}\right)+$ $\phi_{j}\left(q_{j}\right)$ can be written as a function $V\left(t-u, \not p, \not p_{c}-m_{c}, q_{k}^{2}-\right.$ $\left.M_{k}^{2}\right)$ with masses $m_{c}(c=a, b)$ and $M_{k}(k=i, j)$ for the baryons and mesons, respectively, the total momentum $p=p_{a}+q_{i}=p_{b}+q_{j}$ and the Mandelstam variables $t=\left(p_{a}-p_{b}\right)^{2}, u=\left(p_{a}-q_{j}\right)^{2}$. It is understood that the offshell terms $\not p_{a}-m_{a}$ have been moved to the right, whereas the $\not p_{b}-m_{b}$ have been moved to the left. We assume that $V$ is analytic in the variable $t-u$. This is certainly the case for all polynomial interactions which are derived from contact interactions. The Taylor expansion of $V$ in $t-u$ reads

$$
\begin{aligned}
V(t-u) & =\sum_{i=0}^{\infty}(t-u)^{i} V_{i} \\
& =V_{0}+(t-u) V_{1}+(t-u)^{2} V_{2}+\ldots,
\end{aligned}
$$

where we have suppressed the dependence of $V$ and $V_{i}$ on $\not p$ and the off-shell pieces $\not p_{c}-m_{c}$ and $p_{k}^{2}-M_{k}^{2}$ for brevity.
In the center-of-mass frame the variable $t-u$ is related to the scattering angle $\theta$ via

$$
t-u=4\left|\boldsymbol{p}_{a}\right|\left|\boldsymbol{p}_{b}\right| \cos \theta+\frac{1}{p^{2}}\left(p_{a}^{2}-q_{i}^{2}\right)\left(p_{b}^{2}-q_{j}^{2}\right),
$$

where $\left|\boldsymbol{p}_{a, b}\right|$ are the moduli of the c.m. three-momenta. We define a set of orthogonal functions $J_{l}$ with $l=0,1,2, \ldots$ (or $s, p, d, \ldots$ ) which are proportional to the Legendre polynomials in the center-of-mass scattering angle with the first few $J_{l}$ given by

$$
\begin{aligned}
& J_{s}=1, \\
& J_{p}=\left|\boldsymbol{p}_{a}\right|\left|\boldsymbol{p}_{b}\right| \cos \theta=\frac{1}{4}(t-u)-\frac{1}{4 p^{2}}\left(p_{a}^{2}-q_{i}^{2}\right)\left(p_{b}^{2}-q_{j}^{2}\right), \\
& J_{d}=\left|\boldsymbol{p}_{a}\right|^{2}\left|\boldsymbol{p}_{b}\right|^{2}\left(\cos ^{2} \theta-\frac{1}{3}\right)=J_{p}^{2}-\frac{1}{3}\left|\boldsymbol{p}_{a}\right|^{2}\left|\boldsymbol{p}_{b}\right|^{2}, \\
& J_{f}=\left|\boldsymbol{p}_{a}\right|^{3}\left|\boldsymbol{p}_{b}\right|^{3}\left(\cos ^{3} \theta-\frac{3}{5} \cos \theta\right)=J_{p}^{3}-\frac{3}{5}\left|\boldsymbol{p}_{a}\right|^{2}\left|\boldsymbol{p}_{b}\right|^{2} J_{p} .
\end{aligned}
$$

The moduli of the three-momenta in the center-of-mass frame can be expressed in terms of Lorentz scalars as

$$
\begin{aligned}
\left|\boldsymbol{p}_{a}\right|^{2} & =\frac{1}{4 p^{2}}\left(p^{2}+q_{i}^{2}-p_{a}^{2}\right)^{2}-q_{i}^{2} \\
\left|\boldsymbol{p}_{b}\right|^{2} & =\frac{1}{4 p^{2}}\left(p^{2}+q_{j}^{2}-p_{b}^{2}\right)^{2}-q_{j}^{2} .
\end{aligned}
$$

The expansion of the amplitude $V$ in $t-u$ can now be reformulated as an expansion in $J_{l}, V=\sum_{l} V_{l} J_{l}$. In order to keep the presentation simple, we restrict ourselves to the Weinberg-Tomozawa interaction from now on. The generalization to more complicated vertex structures is straightforward. For the Weinberg-Tomozawa term $V$ does not depend on $t-u$,

$$
V=V_{0}=V_{s}
$$

and the division of $V$ into on- and off-shell pieces can be conveniently accomplished by employing the matrix notation (for the reaction $a i \rightarrow b j$ )

$$
\begin{aligned}
V_{s}^{b j, a i} & =\boldsymbol{u}_{b j}^{T} \breve{V}_{s}^{b j, a i} \boldsymbol{u}_{a i}, \\
\boldsymbol{u}_{a i}^{T}\left(\not p_{a}\right) & =\left(1, \not p_{a}-m_{a}\right), \\
\boldsymbol{u}_{b j}^{T}\left(\not p_{b}\right) & =\left(1, \not p_{b}-m_{b}\right), \\
\breve{V}_{s}^{b j, a i}(\not p) & =g^{b j, a i}\left(\begin{array}{cc}
2 \not p-m_{a}-m_{b} & -1 \\
-1 & 0
\end{array}\right),
\end{aligned}
$$

where $g^{b j, a i}$ is the Weinberg-Tomozawa coupling for the channels under consideration as defined in eq. (2.6). The amplitude $V$ is utilized as the interaction kernel in the Bethe-Salpeter equation for the scattering matrix $T, c f$. eq. (2.9),

$$
\begin{aligned}
& T\left(\phi_{j}, \phi_{i} ; p\right)=V\left(\not q_{j}, q_{i}\right) \\
& \quad+\int \frac{d^{d} l}{(2 \pi)^{d}} V\left(\not_{j}, l\right) i S(\not p-\not) \Delta(l) T\left(\not, \phi_{i} ; p\right) .
\end{aligned}
$$


For the decomposition into on- and off-shell pieces we make the same ansatz for the partial wave decomposition of the BSE solution $T$ as for $V$ in eq. A.6.

$$
T_{s}^{b j, a i}=\boldsymbol{u}_{b j}^{T} \breve{T}_{s}^{b j, a i} \boldsymbol{u}_{a i}
$$

This yields a BSE for $\breve{T}_{s}$ which is a matrix equation in the combined channel and off-shell space

$$
\breve{T}_{s}=\breve{V}_{s}+\breve{V}_{s} \breve{G}_{s} \breve{T}_{s}
$$

with a matrix $\breve{G}_{s}$ which is diagonal in channel space with off-shell submatrices

$$
\breve{G}_{s}^{c k, c k}=\int \frac{d^{d} l}{(2 \pi)^{d}} \boldsymbol{u}_{c k}(l) i S(l) \Delta(p-l) \boldsymbol{u}_{c k}^{T}(l) .
$$

Hence, the integral expression in the BSE factorizes also without putting the interaction kernel $V$ and the solution $T$ on-shell. In order to solve this equation by matrix inversion, it is most convenient to decompose the matrices $\breve{T}_{s}$, $\breve{V}_{s}$, and $\breve{G}_{s}$ into a Dirac scalar and a piece proportional to $\not p$ (see also sect. 2).

\section{B Loop Integrals}

The tadpole integral is given by

$$
\begin{aligned}
I_{\mathrm{M}}^{b j, a i} & =\int \frac{d^{d} l}{(2 \pi)^{d}} \frac{i \delta^{b a} \delta^{j i}}{l^{2}-M_{j}^{2}+i 0^{+}} \\
& =\left(2 M_{j}^{2} \bar{\lambda}+\frac{M_{j}^{2}}{8 \pi^{2}} \ln \left(\frac{\mathrm{M}}{\mu}\right)\right) \delta^{b a} \delta^{j i} .
\end{aligned}
$$

Here $\mu$ is the scale of dimensional regularization and

$$
\bar{\lambda}=\frac{\mu^{d-4}}{16 \pi^{2}}\left(\frac{1}{d-4}-\frac{1}{2}\left[\ln (4 \pi)+\Gamma^{\prime}(1)+1\right]\right) .
$$

In practice, we will use the $\overline{M S}$ renormalization scheme, i.e. terms proportional $\bar{\lambda}$ will be dropped from all expressions. In the above result, we have neglected terms of $O(4-d)$ since we are interested in the limit $d \rightarrow 4$. The diagonal entries in the matrix $I_{\mathrm{M}}$ of eq. (2.13) are given by the above expression for the tadpole integral, where $M$ is given by the meson mass of the respective channel. Moreover, the scale $\mu$ can vary between the different channels. We shall also define a diagonal matrix $I_{\mathrm{B}}$ with entries given by tadpole integrals where $M_{j}$ is replaced by the baryon mass $m_{a}$ of each channel.

In the following, we will leave out the $+i 0^{+}$prescription in the loop integrals for brevity. The fundamental scalar loop integral reads

$$
\begin{aligned}
I_{0}(p)= & \int \frac{d^{d} l}{(2 \pi)^{d}} \frac{i}{\left[(p-l)^{2}-m^{2}\right]\left[l^{2}-M^{2}\right]} \\
= & \frac{1}{16 \pi^{2}}\left\{-1+\ln \left(\frac{m^{2}}{\mu^{2}}\right)\right. \\
& +\frac{M^{2}-m^{2}+p^{2}}{2 p^{2}} \ln \left(\frac{M^{2}}{m^{2}}\right) \\
& \left.-\frac{4|\mathbf{q}|}{\sqrt{p^{2}}} \operatorname{artanh}\left(\frac{2|\mathbf{q}| \sqrt{p^{2}}}{(m+M)^{2}-p^{2}}\right)\right\},
\end{aligned}
$$

where

$$
|\mathbf{q}|=\frac{\sqrt{\left(p^{2}-(m+M)^{2}\right)\left(p^{2}-(m-M)^{2}\right)}}{2 \sqrt{p^{2}}}
$$

is equal to the modulus of the center-of-mass three momentum for a system with particle masses $m$ and $M$, and $p^{2}$ the total invariant energy squared of the system.

It is useful to define a diagonal matrix $I_{\mathrm{MB}}(p)$, with elements given by the above loop integral $I_{0}$ of eq. (B.2), where $m$ is the baryon mass $m_{a}$ and $M$ is the meson mass $M_{i}$ of the respective channels. Similarly, we will use matrices $I_{\mathrm{BB}}$ and $I_{\mathrm{MM}}$, where $m$ and $M$ are both given by either the baryon mass $m_{a}$ (for $I_{\mathrm{BB}}$ ) or by $M_{i}$ (for $I_{\mathrm{MM}}$ ), respectively. In the same manner we define

$I_{\mathrm{MBB}}^{b j, a i}=\int \frac{d^{d} l}{(2 \pi)^{d}} \frac{i \delta^{b a} \delta^{j i}}{\left[(p-l)^{2}-m_{a}^{2}\right]\left[\left(p_{1}-l\right)^{2}-m_{a}^{2}\right]\left[l^{2}-M_{i}^{2}\right]}$.

An analogous definition applies for $I_{\mathrm{MMB}}$. Only the diagonal elements are needed here, since the coupling of the photon does not alter the meson-baryon channel. The loop integrals occurring in $I_{\mathrm{MBB}}$ and $I_{\mathrm{MMB}}$ can be expressed in terms of Spence functions [47]. In the course of the calculation, loop integrals with a tensor structure in the integrand are also encountered. For example,

$$
\int \frac{d^{d} l}{(2 \pi)^{d}} \frac{i \delta^{b a} \delta^{j i} l^{\mu}}{\left[(p-l)^{2}-m_{a}^{2}\right]\left[l^{2}-M_{i}^{2}\right]}=p^{\mu}\left[I_{\mathrm{MB}}^{(1)}\right]^{b j, a i}
$$

with

$$
\left[I_{\mathrm{MB}}^{(1)}\right]^{b j, a i}=\frac{1}{2 p^{2}}\left[\left(p^{2}+M_{i}^{2}-m_{a}^{2}\right) I_{\mathrm{MB}}^{b j, a i}+I_{\mathrm{B}}^{b j, a i}-I_{\mathrm{M}}^{b j, a i}\right] .
$$

The last expression is simplified for equal masses in the propagators, so that, e.g., (in matrix notation)

$$
\int \frac{d^{d} l}{(2 \pi)^{d}} i l^{\mu} \Delta(p-l) \Delta(l)=\frac{p^{\mu}}{2} I_{\mathrm{MM}}(p) .
$$

We also need

$$
\int \frac{d^{d} l}{(2 \pi)^{d}} i l^{\mu} l^{\nu} \Delta(p-l) \Delta(l)=g^{\mu \nu} I_{\mathrm{MM}}^{(a)}(p)+\frac{p^{\mu} p^{\nu}}{p^{2}} I_{\mathrm{MM}}^{(b)}(p),
$$

where the coefficients of the tensor structures are given by

$$
\begin{aligned}
& (d-1) I_{\mathrm{MM}}^{(a)}(p)=\left(M^{2}-\frac{1}{4} p^{2}\right) I_{\mathrm{MM}}(p)+\frac{1}{2} I_{\mathrm{M}}, \\
& (d-1) I_{\mathrm{MM}}^{(b)}(p)=\left(\frac{d}{4} p^{2}-M^{2}\right) I_{\mathrm{MM}}(p)+\left(\frac{d}{2}-1\right) I_{\mathrm{M}} .
\end{aligned}
$$

Here we have employed the meson mass matrix

$$
M^{b j, a i}=\delta^{b a} \delta^{j i} M_{j} .
$$

defined in analogy to the baryon mass matrix $m$, and the term $p^{2}$ in the last two equations is of course to be understood as being multiplied by the identity matrix in channel space. 
The matrix $G$ of eq. (2.14) can be given in terms of scalar loop integrals already defined:

$G(p)=\frac{\not p}{2 p^{2}}\left(\left[p^{2}-M^{2}+m^{2}\right] I_{\mathrm{MB}}(p)+I_{\mathrm{M}}-I_{\mathrm{B}}\right)+m I_{\mathrm{MB}}(p)$.

Integrals with a vector or tensor structure in the numerator will also be required in the case of three propagators. In particular, two of the three masses in the propagators will be equal, as already mentioned above. In the following, we will consider the case MBB, noting that the other case (MMB) is then given simply by interchanging $m$ and $M$. First,

$$
\begin{aligned}
& \int \frac{d^{d} l}{(2 \pi)^{d}} \frac{i \delta^{b a} \delta^{j i} l^{\mu}}{\left[(p-l)^{2}-m_{a}^{2}\right]\left[\left(p_{1}-l\right)^{2}-m_{a}^{2}\right]\left[l^{2}-M_{j}^{2}\right]} \\
& =A^{b j, a i}\left(p_{1}, p\right)\left(p_{1}+p\right)^{\mu}+B^{b j, a i}\left(p_{1}, p\right)\left(p_{1}-p\right)^{\mu} .
\end{aligned}
$$

The channel matrices $A$ and $B$ defined by this equation read

$$
\begin{aligned}
A & =\frac{1}{2 D}\left\{\left(4 \bar{M}^{2}-\frac{\Delta p^{4}}{k^{2}}\right) I_{\mathrm{MBB}}+2 I_{\mathrm{BB}}(k)\right. \\
& \left.-\left(1-\frac{\Delta p^{2}}{k^{2}}\right) I_{\mathrm{MB}}\left(p_{1}\right)-\left(1+\frac{\Delta p^{2}}{k^{2}}\right) I_{\mathrm{MB}}(p)\right\}, \\
B & =\frac{\Delta p^{2}}{2 k^{2} D}\left\{\left(4 \bar{M}^{2}+k^{2}-4 \bar{p}^{2}\right) I_{\mathrm{MBB}}+2 I_{\mathrm{BB}}(k)\right. \\
& -\left(1-\frac{4 \bar{p}^{2}-k^{2}}{\Delta p^{2}}\right) I_{\mathrm{MB}}\left(p_{1}\right) \\
& \left.-\left(1+\frac{4 \bar{p}^{2}-k^{2}}{\Delta p^{2}}\right) I_{\mathrm{MB}}(p)\right\} .
\end{aligned}
$$

Here we have used the following notation:

$$
\begin{aligned}
\bar{p}^{2} & =\frac{p_{1}^{2}+p^{2}}{2}, \\
\bar{M}^{2} & =\frac{1}{2}\left(\bar{p}^{2}+M^{2}-m^{2}\right), \\
\Delta p^{2} & =p^{2}-p_{1}^{2}, \\
k & =p-p_{1}, \\
D & =4 \bar{p}^{2}-k^{2}-\frac{\Delta p^{4}}{k^{2}},
\end{aligned}
$$

and the abbreviation $\Delta p^{4} \equiv\left(\Delta p^{2}\right)^{2}$. The loop integral with three propagators and tensor structure is given by

$$
\begin{aligned}
I_{\Delta}^{\mu \nu} & =\int \frac{d^{d} l}{(2 \pi)^{d}} \frac{i \delta^{b a} \delta^{j i} l^{\mu} l^{\nu}}{\left[(p-l)^{2}-m_{a}^{2}\right]\left[\left(p_{1}-l\right)^{2}-m_{a}^{2}\right]\left[l^{2}-M_{j}^{2}\right]} \\
& =C_{1}^{b j, a i} g^{\mu \nu} \\
& +C_{2}^{b j, a i}\left(p_{1}+p\right)^{\mu}\left(p_{1}+p\right)^{\nu}+C_{3}^{b j, a i}\left(p_{1}-p\right)^{\mu}\left(p_{1}-p\right)^{\nu} \\
& +C_{4}^{b j, a i}\left(\left(p_{1}+p\right)^{\mu}\left(p_{1}-p\right)^{\nu}+\left(p_{1}+p\right)^{\nu}\left(p_{1}-p\right)^{\mu}\right) .
\end{aligned}
$$

The coefficient matrices $C_{i}$ read as follows:

$$
C_{1}=\frac{1}{d-2}\left\{M^{2} I_{\mathrm{MBB}}+\frac{1}{2} I_{\mathrm{BB}}(k)-2 \bar{M}^{2} A+\frac{\Delta p^{2}}{2} B\right\},
$$

$$
\begin{aligned}
C_{2} & =\frac{1}{k^{2} D}\left\{k^{2}\left(M^{2} I_{\mathrm{MBB}}+I_{\mathrm{BB}}(k)\right)\right. \\
& +\frac{\Delta p^{2}}{2}\left(k^{2} B-\Delta p^{2} A\right)-\frac{k^{2}}{4}\left(I_{\mathrm{MB}}^{(1)}\left(p_{1}\right)+I_{\mathrm{MB}}^{(1)}(p)\right) \\
& \left.+\frac{\Delta p^{2}}{4}\left(I_{\mathrm{MB}}^{(1)}\left(p_{1}\right)-I_{\mathrm{MB}}^{(1)}(p)\right)-(d-1) k^{2} C_{1}\right\}, \quad(\mathrm{B} .22) \\
C_{3} & =\frac{1}{k^{2} D}\left\{\left(4 \bar{p}^{2}-k^{2}\right)\left(M^{2} I_{\mathrm{MBB}}+\frac{1}{2} I_{\mathrm{BB}}(k)\right)\right. \\
& -2 \bar{M}^{2}\left(\left(4 \bar{p}^{2}-k^{2}\right) A-\Delta p^{2} B\right) \\
& +\frac{1}{4}\left(4 \bar{p}^{2}-\Delta p^{2}-k^{2}\right) I_{\mathrm{MB}}^{(1)}\left(p_{1}\right) \\
& \left.+\frac{1}{4}\left(4 \bar{p}^{2}+\Delta p^{2}-k^{2}\right) I_{\mathrm{MB}}^{(1)}(p)-(d-1)\left(4 \bar{p}^{2}-k^{2}\right) C_{1}\right\}, \\
C_{4} & =\frac{1}{k^{2} D}\left\{\Delta p^{2}\left(M^{2} I_{\mathrm{MBB}}+I_{\mathrm{BB}}(k)\right)\right. \\
& -\frac{\Delta p^{2}}{2}\left(\left(4 \bar{p}^{2}-k^{2}\right) A-\Delta p^{2} B\right) \\
& +\frac{1}{4}\left(4 \bar{p}^{2}-\Delta p^{2}-k^{2}\right) I_{\mathrm{MB}}^{(1)}\left(p_{1}\right) \\
& \left.-\frac{1}{4}\left(4 \bar{p}^{2}+\Delta p^{2}-k^{2}\right) I_{\mathrm{MB}}^{(1)}(p)-(d-1) \Delta p^{2} C_{1}\right\} .
\end{aligned}
$$

$A$ and $B$ are given in eqs. (B.13) and (B.14), while $I_{\mathrm{MB}}^{(1)}$ is defined in eq. (B.6). Note that in the limit $d \rightarrow 4, C_{1}$ will aquire an additional finite contribution due to the divergent terms in the loop integrals. In particular,

$$
\begin{aligned}
C_{1} & \rightarrow C_{1}(d=4)-\frac{1}{64 \pi^{2}}, \\
(d-1) C_{1} & \rightarrow 3 C_{1}(d=4)+\frac{1}{32 \pi^{2}} .
\end{aligned}
$$

As already mentioned, the results for the MMB case can be obtained from the above formulae by interchanging $m \leftrightarrow M$. The corresponding coefficients will be denoted by $\tilde{A}, \tilde{B}$ and $\tilde{C}_{i}$. Finally, the preceding results can be used to derive the decompositions

$$
\begin{aligned}
I_{F}= & \int \frac{d^{d} l}{(2 \pi)^{d}} i S(\not p-\not) e Q_{\mathrm{B}} \gamma^{\mu} S(\not p p-\not) \Delta(l) \\
= & \gamma^{\mu} F_{1}+\not p \gamma^{\mu} F_{2}+\gamma^{\mu} \not p_{1} F_{3}+\not p \gamma^{\mu} \not p_{1} F_{4}+p^{\mu} F_{5} \\
& +p_{1}^{\mu} F_{6}+p^{\mu} \not p F_{7}+p^{\mu} \not p_{1} F_{8}+p_{1}^{\mu} \not p F_{9}+p_{1}^{\mu} p p_{1} F_{10}
\end{aligned}
$$

and

$$
\begin{aligned}
I_{\tilde{F}}= & \int \frac{d^{d} l}{(2 \pi)^{d}} i \Delta(p-l) e Q_{\mathrm{M}}\left(p+p_{1}-2 l\right)^{\mu} \Delta\left(p_{1}-l\right) S(l) \\
= & \gamma^{\mu} \tilde{F}_{1}+\not p \gamma^{\mu} \tilde{F}_{2}+\gamma^{\mu} p p_{1} \tilde{F}_{3}+\not p \gamma^{\mu} p \tilde{F}_{1} \tilde{F}_{4}+p^{\mu} \tilde{F}_{5} \\
& +p_{1}^{\mu} \tilde{F}_{6}+p^{\mu} \not p \tilde{F}_{7}+p^{\mu} \not p \tilde{F}_{8}+p_{1}^{\mu} \not p \tilde{F}_{9}+p_{1}^{\mu} \not p \tilde{F}_{10},
\end{aligned}
$$


where the coefficients of the Lorentz structures are given by

$$
\begin{aligned}
F_{1}= & e Q_{\mathrm{B}}\left(2 C_{1}+\left(m^{2}-M^{2}\right) I_{\mathrm{MBB}}-I_{\mathrm{BB}}(k)\right. \\
& \left.+p_{1}^{2}(A+B)+p^{2}(A-B)\right), \\
F_{2}= & e Q_{\mathrm{B}} m I_{\mathrm{MBB}}, \\
F_{3}= & F_{2} \\
F_{4}= & e Q_{\mathrm{B}}\left(I_{\mathrm{MBB}}-2 A\right), \\
F_{5}= & 2 e Q_{\mathrm{B}} m(B-A), \\
F_{6}= & -2 e Q_{\mathrm{B}} m(B+A), \\
F_{7}= & 2 e Q_{\mathrm{B}}\left(B-A+C_{2}+C_{3}-2 C_{4}\right), \\
F_{8}= & 2 e Q_{\mathrm{B}}\left(C_{2}-C_{3}\right), \\
F_{9}= & F_{8} \\
F_{10}= & 2 e Q_{\mathrm{B}}\left(C_{2}+C_{3}+2 C_{4}-A-B\right), \\
\tilde{F}_{1}= & -2 e Q_{\mathrm{M}} \tilde{C}_{1}, \\
\tilde{F}_{2}= & \tilde{F}_{3}=\tilde{F}_{4}=0, \\
\tilde{F}_{5}= & e Q_{\mathrm{M}}\left(m I_{\mathrm{MMB}}+2 m(\tilde{B}-\tilde{A})\right), \\
\tilde{F}_{6}= & e Q_{\mathrm{M}}\left(m I_{\mathrm{MMB}}-2 m(\tilde{B}+\tilde{A})\right), \\
\tilde{F}_{7}= & e Q_{\mathrm{M}}\left(\tilde{A}-\tilde{B}-2\left(\tilde{C}_{2}+\tilde{C}_{3}-2 \tilde{C}_{4}\right)\right), \\
\tilde{F}_{8}= & e Q_{\mathrm{M}}\left(\tilde{A}+\tilde{B}-2\left(\tilde{C}_{2}-\tilde{C}_{3}\right)\right), \\
\tilde{F}_{9}= & e Q_{\mathrm{M}}\left(\tilde{A}-\tilde{B}-2\left(\tilde{C}_{2}-\tilde{C}_{3}\right)\right), \\
\tilde{F}_{10}= & e Q_{\mathrm{M}}\left(\tilde{A}+\tilde{B}-2\left(\tilde{C}_{2}+\tilde{C}_{3}+2 \tilde{C}_{4}\right)\right) .
\end{aligned}
$$

The following relations are helpful, e.g. when checking gauge invariance:

$$
\begin{aligned}
G_{1}(p)= & \bar{p}^{2} F_{4}-\frac{\Delta p^{2}}{2}\left(F_{4}+F_{7}+F_{9}\right) \\
& +\frac{k^{2}}{2}\left(F_{9}-F_{7}\right)-F_{1} \\
= & \frac{k^{2}}{2}\left(\tilde{F}_{9}-\tilde{F}_{7}\right)-\frac{\Delta p^{2}}{2}\left(\tilde{F}_{9}+\tilde{F}_{7}\right)-\tilde{F}_{1}, \\
G_{1}\left(p_{1}\right)= & \bar{p}^{2} F_{4}+\frac{\Delta p^{2}}{2}\left(F_{4}+F_{8}+F_{10}\right) \\
& +\frac{k^{2}}{2}\left(F_{8}-F_{10}\right)-F_{1} \\
= & \frac{k^{2}}{2}\left(\tilde{F}_{8}-\tilde{F}_{10}\right)+\frac{\Delta p^{2}}{2}\left(\tilde{F}_{8}+\tilde{F}_{10}\right)-\tilde{F}_{1},
\end{aligned}
$$

and also

$$
\begin{aligned}
G_{0}(p)-G_{0}\left(p_{1}\right) & =\frac{k^{2}}{2}\left(F_{6}-F_{5}\right)-\frac{\Delta p^{2}}{2}\left(2 F_{2}+F_{5}+F_{6}\right) \\
& =\frac{k^{2}}{2}\left(\tilde{F}_{6}-\tilde{F}_{5}\right)-\frac{\Delta p^{2}}{2}\left(\tilde{F}_{5}+\tilde{F}_{6}\right) .
\end{aligned}
$$

As a further check of the calculation we have utilized the routines provided by the FeynCalc package [48].

\section{Decomposition of the amplitudes}

We start with the amplitudes of eqs. (3.10)-(3.12).

$$
S_{s}^{\mu}=\left(\not \not p S_{s}^{\phi \not p \gamma}+\not \phi S_{s}^{\phi \gamma \gamma}+\not p S_{s}^{\not p \gamma}+S_{s}^{\gamma}\right) \gamma^{\mu} \gamma_{5}
$$

with

$$
\begin{aligned}
S_{s}^{\phi p \phi \gamma} & =\frac{e}{m_{p}^{2}-s}\left(\Gamma_{2}(p)-m_{p} \Gamma_{1}(p)\right), \\
S_{s}^{\phi \phi \gamma} & =\frac{e}{m_{p}^{2}-s}\left(s \Gamma_{1}(p)-m_{p} \Gamma_{2}(p)\right), \\
S_{s}^{\not p \gamma} & =\frac{e}{m_{p}^{2}-s}\left(\Gamma_{4}(p)-m_{p} \Gamma_{3}(p)\right), \\
S_{s}^{\gamma} & =\frac{e}{m_{p}^{2}-s}\left(s \Gamma_{3}(p)-m_{p} \Gamma_{4}(p)\right) .
\end{aligned}
$$

Here, $m_{p}$ is the proton mass and $s \equiv p^{2}=\left(p_{1}+k\right)^{2}$. Moreover,

$$
S_{u}^{\mu}=q^{\mu} \gamma_{5} S_{u}^{q}+\not q \gamma^{\mu} \gamma_{5} S_{u}^{q \gamma}+\gamma^{\mu} \gamma_{5} S_{u}^{\gamma}
$$

with

$$
\begin{aligned}
S_{u}^{q}= & -2 S_{u}^{q \gamma}=\frac{2 e Q_{\mathrm{B}}}{u-m^{2}} Y_{3}, \\
S_{u}^{\gamma}= & \frac{e Q_{\mathrm{B}}}{m^{2}-u}\left(\Gamma_{1}\left(p_{1}\right) m_{p}\left(u-m_{p}^{2}\right)-\Gamma_{2}\left(p_{1}\right)\left(u-m_{p}^{2}\right)\right. \\
& \left.+\left(m_{p}^{2}-m_{p} m\right) \Gamma_{3}\left(p_{1}\right)+\left(m-m_{p}\right) \Gamma_{4}\left(p_{1}\right)\right),
\end{aligned}
$$

and

$$
\begin{aligned}
S_{t}^{\mu}= & \left(p^{\mu} S_{t}^{p}+p_{1}^{\mu} S_{t}^{p_{1}}+q^{\mu} S_{t}^{q}+\not q q^{\mu} S_{t}^{q q}+\not p q^{\mu} S_{t}^{\not p q}\right. \\
& \left.+\not p p^{\mu} S_{t}^{q p}+\not p p^{\mu} S_{t}^{\not p p}+\not p p_{1}^{\mu} S_{t}^{q p_{1}}+\not p p_{1}^{\mu} S_{t}^{\not p p_{1}}\right) \gamma_{5},
\end{aligned}
$$

with

$$
\begin{aligned}
S_{t}^{p} & =\frac{e Q_{\mathrm{M}}}{t-M^{2}} Y_{1}, & S_{t}^{p_{1}} & =-S_{t}^{p}, & S_{t}^{q} & =-2 S_{t}^{p}, \\
S_{t}^{\phi q} & =-\frac{2 e Q_{\mathrm{M}}}{t-M^{2}} Y_{2}, & S_{t}^{\phi q} & =-S_{t}^{q q}, & S_{t}^{q p} & =\frac{e Q_{\mathrm{M}}}{t-M^{2}} Y_{2}, \\
S_{t}^{\not p} & =-S_{t}^{q p}, & S_{t}^{q p} & =-S_{t}^{q p}, & S_{t}^{\phi p_{1}} & =+S_{t}^{q p} .
\end{aligned}
$$

The foregoing results deserve some comments. We use the abbreviations

$$
\begin{aligned}
Y_{1}= & m_{p}^{2} \Gamma_{1}\left(p_{1}\right)-m_{p}\left(\Gamma_{2}\left(p_{1}\right)+\Gamma_{3}\left(p_{1}\right)\right)+\Gamma_{4}\left(p_{1}\right), \\
Y_{2}= & \Gamma_{2}\left(p_{1}\right)-m_{p} \Gamma_{1}\left(p_{1}\right) \\
Y_{3}= & \left(m_{p}^{2}+m_{p} m\right) \Gamma_{1}\left(p_{1}\right)-\left(m+m_{p}\right) \Gamma_{2}\left(p_{1}\right) \\
& -m_{p} \Gamma_{3}\left(p_{1}\right)+\Gamma_{4}\left(p_{1}\right)
\end{aligned}
$$

Furthermore, we have anticipated that the full amplitude will be multiplied by spinors, so we set $\not p_{1} \gamma_{5} \rightarrow-m_{p} \gamma_{5}$. The Mandelstam variables $u$ and $t$ are understood to be diagonal matrices in channel space and are defined via the center-of-mass (c.m.) relations

$$
\begin{aligned}
t & =M^{2}+k^{2}-2 E_{k} E_{q}+2|\mathbf{k}||\mathbf{q}| \cos \theta, \\
u & =k^{2}+m_{p}^{2}+m^{2}+M^{2}-s-t,
\end{aligned}
$$

where $|\mathbf{q}|$ and $|\mathbf{k}|$ are the moduli of the c.m. three-momenta of the outgoing meson and the incoming photon, respectively (see eq. (B.3)), $\theta$ is the scattering angle in the c.m. 
B. Borasoy et al.: A gauge invariant chiral unitary framework for kaon photo- and electroproduction on the proton

frame, and the c.m. energies are given by

$$
\begin{aligned}
& E_{k}=\sqrt{|\mathbf{k}|^{2}+k^{2}} \\
& E_{q}=\sqrt{|\mathbf{q}|^{2}+M^{2}}
\end{aligned}
$$

Of course, multiplication of scalar quantities (such as $k^{2}$ or $m_{p}$ ) by the unit matrix in channel space is implied where necessary and fractions involving matrix-valued denominators denote matrix inversions.

Now we come to the graphs where the photon couples to internal meson or baryon lines. The decomposition of $S_{\mathrm{B}}^{\mu}$ reads

$$
\begin{aligned}
S_{\mathrm{B}}^{\mu}= & \left(\gamma^{\mu} S_{\mathrm{B}}^{\gamma}+p^{\mu} S_{\mathrm{B}}^{p}+p_{1}^{\mu} S_{\mathrm{B}}^{p_{1}}+\not q \gamma^{\mu} S_{\mathrm{B}}^{q \gamma}\right. \\
& +\not p \gamma^{\mu} S_{\mathrm{B}}^{\not p \gamma}+\not p p \gamma^{\mu} S_{\mathrm{B}}^{q \not p \gamma}+\not p p^{\mu} S_{\mathrm{B}}^{q p}+\not p p^{\mu} S_{\mathrm{B}}^{\not p p} \\
& \left.+\not \not p p^{\mu} S_{\mathrm{B}}^{q \not p p}+\not p p_{1}^{\mu} S_{\mathrm{B}}^{q p_{1}}+\not p p_{1}^{\mu} S_{\mathrm{B}}^{\not p p_{1}}+\not p \not p p_{1}^{\mu} S_{\mathrm{B}}^{q \not p p_{1}}\right) \gamma_{5}
\end{aligned}
$$

with

$$
\begin{aligned}
& S_{\mathrm{B}}^{\gamma}=m_{p}\left[T_{8} F_{3}-T_{5} m F_{3}-T_{5} e Q_{\mathrm{B}} G_{1}\left(p_{1}\right)\right. \\
& \left.+s\left(T_{3} F_{3}+T_{5} F_{4}+T_{7} F_{4}-T_{3} m F_{4}\right)\right] Y_{3} \\
& -T_{8} F_{1} Y_{3}+T_{5} m F_{1} Y_{3}+T_{5} e Q_{\mathrm{B}} G_{0}\left(p_{1}\right) Y_{3} \\
& -T_{5} e Q_{\mathrm{B}} I_{\mathrm{M}} Y_{2}+T_{8} G_{0}(p) e Q_{\mathrm{B}} Y_{2}-T_{5} m G_{0}(p) e Q_{\mathrm{B}} Y_{2} \\
& -s\left(T_{3} F_{1} Y_{3}+T_{5} F_{2} Y_{3}+T_{7} F_{2} Y_{3}-T_{3} G_{0}(p) e Q_{\mathrm{B}} Y_{2}\right. \\
& -T_{3} m F_{2} Y_{3}-T_{5} G_{1}(p) e Q_{\mathrm{B}} Y_{2} \\
& \left.-T_{7} G_{1}(p) e Q_{\mathrm{B}} Y_{2}+T_{3} m G_{1}(p) e Q_{\mathrm{B}} Y_{2}\right), \\
& S_{\mathrm{B}}^{p}=m_{p}\left[T_{8} F_{8} Y_{3}-T_{5} m F_{8} Y_{3}+s T_{3} F_{8} Y_{3}\right] \\
& -T_{8} F_{5} Y_{3}+T_{5} m F_{5} Y_{3} \\
& -s\left(T_{3} F_{5} Y_{3}+T_{5} F_{7} Y_{3}+T_{7} F_{7} Y_{3}-T_{3} m F_{7} Y_{3}\right) \text {, } \\
& S_{\mathrm{B}}^{p_{1}}=m_{p}\left[T_{8} F_{10} Y_{3}-T_{5} m F_{10} Y_{3}+s T_{3} F_{10} Y_{3}\right]-T_{8} F_{6} Y_{3} \\
& +T_{5} m F_{6} Y_{3}-s\left(T_{3} F_{6}+T_{5} F_{9}+T_{7} F_{9}-T_{3} m F_{9}\right) Y_{3}, \\
& S_{\mathrm{B}}^{\phi q \gamma}=m_{p}\left[T_{6} F_{3}-T_{2} m F_{3}-T_{2} e Q_{\mathrm{B}} G_{1}\left(p_{1}\right)\right. \\
& \left.+s\left(T_{1} F_{3}+T_{2} F_{4}+T_{4} F_{4}-T_{1} m F_{4}\right)\right] Y_{3} \\
& -T_{6} F_{1} Y_{3}+T_{2} m F_{1} Y_{3}+T_{2} e Q_{\mathrm{B}} G_{0}\left(p_{1}\right) Y_{3} \\
& -T_{2} e Q_{\mathrm{B}} I_{\mathrm{M}} Y_{2}+T_{6} G_{0}(p) e Q_{\mathrm{B}} Y_{2}-T_{2} m G_{0}(p) e Q_{\mathrm{B}} Y_{2} \\
& -s\left(T_{1} F_{1} Y_{3}+T_{2} F_{2} Y_{3}+T_{4} F_{2} Y_{3}-T_{1} G_{0}(p) e Q_{\mathrm{B}} Y_{2}\right. \\
& -T_{1} m F_{2} Y_{3}-T_{2} G_{1}(p) e Q_{\mathrm{B}} Y_{2}-T_{4} G_{1}(p) e Q_{\mathrm{B}} Y_{2} \\
& \left.+T_{1} m G_{1}(p) e Q_{\mathrm{B}} Y_{2}\right) \text {, } \\
& S_{\mathrm{B}}^{\not p \gamma}=m_{p}\left[T_{5} F_{3}+T_{7} F_{3}+T_{8} F_{4}-T_{3} m F_{3}\right. \\
& \left.-T_{3} e Q_{\mathrm{B}} G_{1}\left(p_{1}\right)-T_{5} m F_{4}+s T_{3} F_{4}\right] Y_{3} \\
& -T_{5} F_{1} Y_{3}-T_{7} F_{1} Y_{3}-T_{8} F_{2} Y_{3}+T_{3} m F_{1} Y_{3} \\
& +T_{3} e Q_{\mathrm{B}} G_{0}\left(p_{1}\right) Y_{3}-T_{3} e Q_{\mathrm{B}} I_{\mathrm{M}} Y_{2} \\
& +T_{5} G_{0}(p) e Q_{\mathrm{B}} Y_{2}+T_{5} m F_{2} Y_{3}+T_{7} G_{0}(p) e Q_{\mathrm{B}} Y_{2} \\
& +T_{8} G_{1}(p) e Q_{\mathrm{B}} Y_{2}-T_{3} m G_{0}(p) e Q_{\mathrm{B}} Y_{2} \\
& -T_{5} m G_{1}(p) e Q_{\mathrm{B}} Y_{2}+s\left(T_{3} G_{1}(p) e Q_{\mathrm{B}} Y_{2}-T_{3} F_{2} Y_{3}\right) \text {, }
\end{aligned}
$$

$$
\begin{aligned}
& S_{\mathrm{B}}^{\not \not p \gamma}=m_{p}\left[T_{2} F_{3}+T_{4} F_{3}+T_{6} F_{4}-T_{1} m F_{3}\right. \\
& \left.-T_{1} e Q_{\mathrm{B}} G_{1}\left(p_{1}\right)-T_{2} m F_{4}+s T_{1} F_{4}\right] Y_{3} \\
& -T_{2} F_{1} Y_{3}-T_{4} F_{1} Y_{3}-T_{6} F_{2} Y_{3} \\
& +T_{1} m F_{1} Y_{3}+T_{1} e Q_{\mathrm{B}} G_{0}\left(p_{1}\right) Y_{3}-T_{1} e Q_{\mathrm{B}} I_{\mathrm{M}} Y_{2} \\
& +T_{2} G_{0}(p) e Q_{\mathrm{B}} Y_{2}+T_{2} m F_{2} Y_{3}+T_{4} G_{0}(p) e Q_{\mathrm{B}} Y_{2} \\
& +T_{6} G_{1}(p) e Q_{\mathrm{B}} Y_{2}-T_{1} m G_{0}(p) e Q_{\mathrm{B}} Y_{2} \\
& -T_{2} m G_{1}(p) e Q_{\mathrm{B}} Y_{2}-s\left(T_{1} F_{2} Y_{3}-T_{1} G_{1}(p) e Q_{\mathrm{B}} Y_{2}\right), \\
& S_{\mathrm{B}}^{q p}=m_{p}\left[T_{6} F_{8}-T_{2} m F_{8}+s T_{1} F_{8}\right] Y_{3} \\
& -T_{6} F_{5} Y_{3}+T_{2} m F_{5} Y_{3} \\
& -s\left(T_{1} F_{5}+T_{2} F_{7}+T_{4} F_{7}-T_{1} m F_{7}\right) Y_{3}, \\
& S_{\mathrm{B}}^{\not p p}=m_{p}\left[T_{5} F_{8}+T_{7} F_{8}-T_{3} m F_{8}\right] Y_{3} \\
& -\left(T_{5} F_{5}+T_{7} F_{5}+T_{8} F_{7}-T_{3} m F_{5}\right. \\
& \left.-T_{5} m F_{7}+s T_{3} F_{7}\right) Y_{3} \text {, } \\
& S_{\mathrm{B}}^{\text {qlpp }}=m_{p}\left[T_{2} F_{8}+T_{4} F_{8}-T_{1} m F_{8}\right] Y_{3} \\
& -\left(T_{2} F_{5}+T_{4} F_{5}+T_{6} F_{7}-T_{1} m F_{5}\right. \\
& \left.-T_{2} m F_{7}+s T_{1} F_{7}\right) Y_{3} \text {, } \\
& S_{\mathrm{B}}^{q p_{1}}=m_{p}\left[T_{6} F_{10}-T_{2} m F_{10}+s T_{1} F_{10}\right] Y_{3} \\
& -T_{6} F_{6} Y_{3}+T_{2} m F_{6} Y_{3} \\
& -s\left(T_{1} F_{6}+T_{2} F_{9}+T_{4} F_{9}-T_{1} m F_{9}\right) Y_{3}, \\
& S_{\mathrm{B}}^{\not p p_{1}}=m_{p}\left[T_{5} F_{10}+T_{7} F_{10}-T_{3} m F_{10}\right] Y_{3} \\
& -\left(T_{5} F_{6}+T_{7} F_{6}+T_{8} F_{9}-T_{3} m F_{6}\right. \\
& \left.-T_{5} m F_{9}+s T_{3} F_{9}\right) Y_{3} \text {, } \\
& S_{\mathrm{B}}^{q \not p p_{1}}=m_{p}\left[T_{2} F_{10}+T_{4} F_{10}-T_{1} m F_{10}\right] Y_{3} \\
& -\left(T_{2} F_{6}+T_{4} F_{6}+T_{6} F_{9}-T_{1} m F_{6}\right. \\
& \left.-T_{2} m F_{9}+s T_{1} F_{9}\right) Y_{3} \text {, }
\end{aligned}
$$

while the decomposition of $S_{\mathrm{M}}^{\mu}$ reads

$$
\begin{aligned}
S_{\mathrm{M}}^{\mu}= & \left(\gamma^{\mu} S_{\mathrm{M}}^{\gamma}+p^{\mu} S_{\mathrm{M}}^{p}+p_{1}^{\mu} S_{\mathrm{M}}^{p_{1}}+\not q \gamma^{\mu} S_{\mathrm{M}}^{q \gamma \gamma}+\not p \gamma^{\mu} S_{\mathrm{M}}^{\not p \gamma}\right. \\
& +\not \not p \gamma^{\mu} S_{\mathrm{M}}^{\phi p p \gamma}+\not p p^{\mu} S_{\mathrm{M}}^{\phi p}+\not p p^{\mu} S_{\mathrm{M}}^{\not p p}+\not \not p p^{\mu} S_{\mathrm{M}}^{\phi \not p p} \\
& \left.+\not p p_{1}^{\mu} S_{\mathrm{M}}^{q p_{1}}+\not p p_{1}^{\mu} S_{\mathrm{M}}^{\not p p_{1}}+\not \not p p_{1}^{\mu} S_{\mathrm{M}}^{q \not p p_{1}}\right) \gamma_{5},
\end{aligned}
$$

with

$$
\begin{aligned}
S_{\mathrm{M}}^{\gamma} & =T_{5} m \tilde{F}_{1} Y_{3}-T_{8} \tilde{F}_{1} Y_{3}-T_{5} e Q_{\mathrm{M}} d_{1} Y_{2}-s T_{3} \tilde{F}_{1} Y_{3} \\
S_{\mathrm{M}}^{p} & =m_{p}\left[T_{8} \tilde{F}_{8} Y_{3}-T_{5} m \tilde{F}_{8} Y_{3}-T_{5} e Q_{\mathrm{M}} d_{2} Y_{2}+s T_{3} \tilde{F}_{8} Y_{3}\right] \\
& -T_{8} \tilde{F}_{5} Y_{3}+T_{5} m \tilde{F}_{5} Y_{3}-s\left(T_{3} \tilde{F}_{5} Y_{3}+T_{5} \tilde{F}_{7} Y_{3}\right. \\
& \left.+T_{7} \tilde{F}_{7} Y_{3}-T_{3} m \tilde{F}_{7} Y_{3}+T_{3} e Q_{\mathrm{M}} d_{2} Y_{2}\right) \\
S_{\mathrm{M}}^{p_{1}} & =m_{p}\left[T_{8} \tilde{F}_{10} Y_{3}-T_{5} m \tilde{F}_{10} Y_{3}+T_{5} e Q_{\mathrm{M}} d_{2} Y_{2}\right. \\
& \left.+s T_{3} \tilde{F}_{10} Y_{3}\right]-T_{8} \tilde{F}_{6} Y_{3}+T_{5} m \tilde{F}_{6} Y_{3} \\
& -s\left(T_{3} \tilde{F}_{6}+T_{5} \tilde{F}_{9}+T_{7} \tilde{F}_{9}-T_{3} m \tilde{F}_{9}\right) Y_{3} \\
& +s T_{3} e Q_{\mathrm{M}} d_{2} Y_{2}, \\
S_{\mathrm{M}}^{\phi \gamma} & =T_{2} m \tilde{F}_{1} Y_{3}-T_{6} \tilde{F}_{1} Y_{3}-s T_{1} \tilde{F}_{1} Y_{3}-T_{2} e Q_{\mathrm{M}} d_{1} Y_{2}, \\
S_{\mathrm{M}}^{\not p \gamma} & =T_{3} m \tilde{F}_{1} Y_{3}-T_{5} \tilde{F}_{1} Y_{3}-T_{7} \tilde{F}_{1} Y_{3}-T_{3} e Q_{\mathrm{M}} d_{1} Y_{2}, \\
S_{\mathrm{M}}^{\phi \not p \gamma} & =T_{1} m \tilde{F}_{1} Y_{3}-T_{2} \tilde{F}_{1} Y_{3}-T_{4} \tilde{F}_{1} Y_{3}-T_{1} e Q_{\mathrm{M}} d_{1} Y_{2},
\end{aligned}
$$




$$
\begin{aligned}
& S_{\mathrm{M}}^{q p}=m_{p}\left[T_{6} \tilde{F}_{8} Y_{3}-T_{2} m \tilde{F}_{8} Y_{3}+s T_{1} \tilde{F}_{8} Y_{3}\right. \\
& \left.-T_{2} e Q_{\mathrm{M}} d_{2} Y_{2}\right]-T_{6} \tilde{F}_{5} Y_{3}+T_{2} m \tilde{F}_{5} Y_{3} \\
& -s\left(T_{1} \tilde{F}_{5}+T_{2} \tilde{F}_{7}+T_{4} \tilde{F}_{7}-T_{1} m \tilde{F}_{7}\right) Y_{3} \\
& -s T_{1} e Q_{\mathrm{M}} d_{2} Y_{2} \\
& S_{\mathrm{M}}^{p p}=m_{p}\left[T_{5} \tilde{F}_{8} Y_{3}+T_{7} \tilde{F}_{8} Y_{3}-T_{3} m \tilde{F}_{8} Y_{3}-T_{3} e Q_{\mathrm{M}} d_{2} Y_{2}\right] \\
& -\left(T_{5} \tilde{F}_{5}+T_{7} \tilde{F}_{5}+T_{8} \tilde{F}_{7}-T_{3} m \tilde{F}_{5}-T_{5} m \tilde{F}_{7}\right) Y_{3} \\
& -s T_{3} \tilde{F}_{7} Y_{3}-T_{5} e Q_{\mathrm{M}} d_{2} Y_{2}, \\
& S_{\mathrm{M}}^{\not \phi p p}=m_{p}\left[T_{2} \tilde{F}_{8} Y_{3}+T_{4} \tilde{F}_{8} Y_{3}-T_{1} m \tilde{F}_{8} Y_{3}-T_{1} e Q_{\mathrm{M}} d_{2} Y_{2}\right] \\
& -\left(T_{2} \tilde{F}_{5}+T_{4} \tilde{F}_{5}+T_{6} \tilde{F}_{7}-T_{1} m \tilde{F}_{5}-T_{2} m \tilde{F}_{7}\right) \\
& -s T_{1} \tilde{F}_{7} Y_{3}-T_{2} e Q_{\mathrm{M}} d_{2} Y_{2}, \\
& S_{\mathrm{M}}^{q p_{1}}=m_{p}\left[T_{6} \tilde{F}_{10} Y_{3}-T_{2} m \tilde{F}_{10} Y_{3}+s T_{1} \tilde{F}_{10} Y_{3}\right. \\
& \left.+T_{2} e Q_{\mathrm{M}} d_{2} Y_{2}\right]-T_{6} \tilde{F}_{6} Y_{3}+T_{2} m \tilde{F}_{6} Y_{3} \\
& +s T_{1} e Q_{\mathrm{M}} d_{2} Y_{2} \\
& -s\left(T_{1} \tilde{F}_{6}+T_{2} \tilde{F}_{9}+T_{4} \tilde{F}_{9}-T_{1} m \tilde{F}_{9}\right) Y_{3}, \\
& S_{\mathrm{M}}^{\not p p_{1}}=m_{p}\left[T_{5} \tilde{F}_{10} Y_{3}+T_{7} \tilde{F}_{10} Y_{3}\right. \\
& \left.-T_{3} m \tilde{F}_{10} Y_{3}+T_{3} e Q_{\mathrm{M}} d_{2} Y_{2}\right] \\
& -\left(T_{5} \tilde{F}_{6}+T_{7} \tilde{F}_{6}+T_{8} \tilde{F}_{9}-T_{3} m \tilde{F}_{6}-T_{5} m \tilde{F}_{9}\right) Y_{3} \\
& -s T_{3} \tilde{F}_{9} Y_{3}+T_{5} e Q_{\mathrm{M}} d_{2} Y_{2}, \\
& S_{\mathrm{M}}^{\phi \phi p p_{1}}=m_{p}\left[T_{2} \tilde{F}_{10} Y_{3}+T_{4} \tilde{F}_{10} Y_{3}\right. \\
& \left.-T_{1} m \tilde{F}_{10} Y_{3}+T_{1} e Q_{\mathrm{M}} d_{2} Y_{2}\right] \\
& -\left(T_{2} \tilde{F}_{6}+T_{4} \tilde{F}_{6}+T_{6} \tilde{F}_{9}-T_{1} m \tilde{F}_{6}-T_{2} m \tilde{F}_{9}\right) Y_{3} \\
& -s T_{1} \tilde{F}_{9} Y_{3}+T_{2} e Q_{\mathrm{M}} d_{2} Y_{2} \text {. }
\end{aligned}
$$

Here we have used the abbreviations $T_{i} \equiv T_{i}(p)$ and

$$
\begin{aligned}
d_{1} & =-2\left[\frac{1}{3}\left(\left(M^{2}-\frac{1}{4} k^{2}\right) I_{\mathrm{MM}}(k)+\frac{1}{2} I_{\mathrm{M}}\right)+\delta\right], \\
d_{2} & =\frac{1}{2} I_{\mathrm{MM}}(k)-\frac{2}{k^{2}}\left[\frac{1}{3}\left(\left(k^{2}-M^{2}\right) I_{\mathrm{MM}}(k)+I_{\mathrm{M}}\right)-\delta\right], \\
\delta & =\frac{1}{48 \pi^{2}}\left[\frac{1}{6} k^{2}-M^{2}\right] .
\end{aligned}
$$

The $T_{i}(p)$ are defined in eq. (2.33), while the $F_{i}$ and $\tilde{F}_{i}$ are defined in eq. (B.27) and (B.28), respectively. The term $\delta$ stems from the limit $d \rightarrow 4$ in eqs. (B.9) and (B.10).

Next we turn to the class of diagrams derived from the 'Kroll-Ruderman'-term (called 'Class 4' in sect. 3):

$$
S_{\mathrm{KR}}^{\mu}=\left(S_{\mathrm{KR}}^{\gamma}+\not q S_{\mathrm{KR}}^{\phi \gamma \gamma}+\not p S_{\mathrm{KR}}^{\not p \gamma}+\not p p S_{\mathrm{KR}}^{\phi \phi p \gamma}\right) \gamma^{\mu} \gamma_{5}
$$

with

$$
\begin{aligned}
S_{\mathrm{KR}}^{\gamma} & =e Q_{\mathrm{M}} \hat{g}+\left[\left(T_{8}-T_{5} m+s T_{3}\right) G_{0}(p)\right. \\
& \left.+s\left(T_{5}+T_{7}-T_{3} m\right) G_{1}(p)-T_{5} I_{\mathrm{M}}\right] e Q_{\mathrm{M}} \hat{g}, \\
S_{\mathrm{KR}}^{\phi \gamma} & =\left[\left(T_{6}-T_{2} m+s T_{1}\right) G_{0}(p)\right. \\
& \left.+s\left(T_{2}+T_{4}-T_{1} m\right) G_{1}(p)-T_{2} I_{\mathrm{M}}\right] e Q_{\mathrm{M}} \hat{g}, \\
S_{\mathrm{KR}}^{\phi \gamma \gamma} & =\left[\left(T_{5}+T_{7}-T_{3} m\right) G_{0}(p)\right. \\
& \left.+\left(s T_{3}+T_{8}-T_{5} m\right) G_{1}(p)-T_{3} I_{\mathrm{M}}\right] e Q_{\mathrm{M}} \hat{g},
\end{aligned}
$$

$$
\begin{aligned}
S_{\mathrm{KR}}^{q \not p \gamma} & =\left[\left(T_{2}+T_{4}-T_{1} m\right) G_{0}(p)\right. \\
& \left.+\left(s T_{1}+T_{6}-T_{2} m\right) G_{1}(p)-T_{1} I_{\mathrm{M}}\right] e Q_{\mathrm{M}} \hat{g} .
\end{aligned}
$$

Here, the first term in the first line is the contribution from the tree graph, and $T_{i} \equiv T_{i}(p)$. The last class of graphs is $S_{W T 1}^{\mu}+S_{W T 2}^{\mu}$, where

$$
S_{W T 1}^{\mu}=\gamma^{\mu} \gamma_{5} S_{W T 1}^{\gamma}
$$

with

$S_{W T 1}^{\gamma}=\left(Q_{\mathrm{M}} g+g Q_{\mathrm{M}}\right)\left(\left(G_{0}\left(p_{1}\right)-m_{p} G_{1}\left(p_{1}\right)\right) Y_{3}-I_{\mathrm{M}} Y_{2}\right)$,

and

$$
S_{W T 2}^{\mu}=\left(S_{W T 2}^{\gamma}+\not S_{W T 2}^{\not \phi \gamma}+\not p S_{W T 2}^{\not p \gamma}+\not \not p S_{W T 2}^{\phi p p \gamma}\right) \gamma^{\mu} \gamma_{5}
$$

with

$$
\begin{aligned}
S_{W T 2}^{\gamma} & =\left[s\left(T_{3} G_{0}(p)+\left(T_{5}+T_{7}-T_{3} m\right) G_{1}(p)\right)\right. \\
& \left.+\left(T_{8}-T_{5} m\right) G_{0}(p)-T_{5} I_{\mathrm{M}}\right] S_{W T 1}^{\gamma}, \\
S_{W T 2}^{q \gamma} & =\left[s\left(T_{1} G_{0}(p)+\left(T_{2}+T_{4}-T_{1} m\right) G_{1}(p)\right)\right. \\
& \left.+\left(T_{6}-T_{2} m\right) G_{0}(p)-T_{2} I_{\mathrm{M}}\right] S_{W T 1}^{\gamma}, \\
S_{W T 2}^{\not p \gamma} & =\left[\left(s T_{3}-T_{5} m+T_{8}\right) G_{1}(p)\right. \\
& \left.+\left(T_{5}+T_{7}-T_{3} m\right) G_{0}(p)-T_{3} I_{\mathrm{M}}\right] S_{W T 1}^{\gamma}, \\
S_{W T 2}^{\phi \not p \gamma} & =\left[\left(s T_{1}-T_{2} m+T_{6}\right) G_{1}(p)\right. \\
& \left.+\left(T_{2}+T_{4}-T_{1} m\right) G_{0}(p)-T_{1} I_{\mathrm{M}}\right] S_{W T 1}^{\gamma} .
\end{aligned}
$$

Adding the contributions of eqs. (C.1)-(C.3) and eqs. (C.9)(C.13), we can decompose the full photoproduction amplitude $\mathcal{M}^{\mu}$ (see eq. (3.21)) as follows:

$$
\begin{aligned}
\mathcal{M}^{\mu} & =\gamma^{\mu} \gamma_{5} \mathcal{M}_{1}+q^{\mu} \gamma_{5} \mathcal{M}_{2}+p^{\mu} \gamma_{5} \mathcal{M}_{3}+p_{1}^{\mu} \gamma_{5} \mathcal{M}_{4} \\
& +\not q \gamma^{\mu} \gamma_{5} \mathcal{M}_{5}+\not p \gamma^{\mu} \gamma_{5} \mathcal{M}_{6}+\not p p \gamma^{\mu} \gamma_{5} \mathcal{M}_{7} \\
& +\not q q^{\mu} \gamma_{5} \mathcal{M}_{8}+\not p q^{\mu} \gamma_{5} \mathcal{M}_{9}+\not p p^{\mu} \gamma_{5} \mathcal{M}_{10} \\
& +\not p p^{\mu} \gamma_{5} \mathcal{M}_{11}+\not \not p p p^{\mu} \gamma_{5} \mathcal{M}_{12}+q p_{1}^{\mu} \gamma_{5} \mathcal{M}_{13} \\
& +\not p p_{1}^{\mu} \gamma_{5} \mathcal{M}_{14}+\not \not p p p_{1}^{\mu} \gamma_{5} \mathcal{M}_{15}
\end{aligned}
$$

This can be simplified, using the Dirac equation and momentum conservation, to arrive at the operator basis given by the $\mathcal{N}_{k}^{\mu}$ commonly used for photoproduction processes, see eq. (D.1). The relation between the corresponding coefficients $B_{k}$ and the functions $\mathcal{M}_{j}$ used in eq. (C.14) is given in eq. (D.2). The decomposition of the amplitudes into the various Dirac structures is obtained by employing the FeynCalc package [48].

\section{Invariant amplitudes}

We consider the reaction

$$
\gamma(k)+p\left(p_{1}, m_{1}\right) \rightarrow B\left(p_{2}, m_{2}\right)+M\left(q, M_{\phi}\right)
$$

and define the Mandelstam variables as usual,

$$
\begin{aligned}
s & =\left(p_{1}+k\right)^{2}, \\
u & =\left(p_{1}-q\right)^{2}, \\
t & =\left(p_{2}-p_{1}\right)^{2} .
\end{aligned}
$$


They obey the constraint

$$
s+t+u=m_{1}^{2}+m_{2}^{2}+M_{\phi}^{2}+k^{2} .
$$

The amplitude can be decomposed as

$$
T_{f i}=i \epsilon_{\mu} \bar{u}_{2} \sum_{k=1}^{8} B_{k} \mathcal{N}_{k}^{\mu} u_{1},
$$

where the operator basis is given by

$$
\begin{array}{lll}
\mathcal{N}_{1}^{\mu}=\gamma_{5} \gamma^{\mu} k, & \mathcal{N}_{2}^{\mu}=2 \gamma_{5} P^{\mu}, & \mathcal{N}_{3}^{\mu}=2 \gamma_{5} q^{\mu}, \\
\mathcal{N}_{4}^{\mu}=2 \gamma_{5} k^{\mu}, & \mathcal{N}_{5}^{\mu}=\gamma_{5} \gamma^{\mu}, & \mathcal{N}_{6}^{\mu}=\gamma_{5} \not k P^{\mu}, \\
\mathcal{N}_{7}^{\mu}=\gamma_{5} \not k k^{\mu}, & \mathcal{N}_{8}^{\mu}=\gamma_{5} \not k q^{\mu} . &
\end{array}
$$

Here, $P=\frac{1}{2}\left(p_{1}+p_{2}\right)$. The relation to the coefficient functions $\mathcal{M}_{j}$ used in eq. (C.14) is

$$
\begin{aligned}
B_{1} & =-\mathcal{M}_{5}-\mathcal{M}_{6}+m_{2} \mathcal{M}_{7} \\
B_{2} & =\frac{1}{2} \mathcal{M}_{3}+\frac{1}{2} \mathcal{M}_{4}+\mathcal{M}_{5}+\mathcal{M}_{6}-m_{2} \mathcal{M}_{7} \\
& -\frac{1}{2}\left(m_{1}+m_{2}\right) \mathcal{M}_{10}-\frac{1}{2} m_{1} \mathcal{M}_{11}+\frac{1}{2}\left(s+m_{1} m_{2}\right) \mathcal{M}_{12} \\
& -\frac{1}{2}\left(m_{1}+m_{2}\right) \mathcal{M}_{13}-\frac{1}{2} m_{1} \mathcal{M}_{14}+\frac{1}{2}\left(s+m_{1} m_{2}\right) \mathcal{M}_{15} \\
B_{3} & =\frac{1}{2} \mathcal{M}_{2}+\frac{1}{4} \mathcal{M}_{3}+\frac{1}{4} \mathcal{M}_{4}+\frac{1}{2} \mathcal{M}_{5}+\frac{1}{2} \mathcal{M}_{6} \\
& -\frac{1}{2} m_{2} \mathcal{M}_{7}-\frac{1}{2}\left(m_{1}+m_{2}\right) \mathcal{M}_{8}-\frac{1}{2} m_{1} \mathcal{M}_{9} \\
& -\frac{1}{4}\left(m_{1}+m_{2}\right) \mathcal{M}_{10}-\frac{1}{4} m_{1} \mathcal{M}_{11}+\frac{1}{4}\left(s+m_{1} m_{2}\right) \mathcal{M}_{12} \\
& -\frac{1}{4}\left(m_{1}+m_{2}\right) \mathcal{M}_{13}-\frac{1}{4} m_{1} \mathcal{M}_{14}+\frac{1}{4}\left(s+m_{1} m_{2}\right) \mathcal{M}_{15} \\
B_{4} & =\frac{1}{4} \mathcal{M}_{3}-\frac{1}{4} \mathcal{M}_{4}+\frac{1}{2} \mathcal{M}_{5}+\frac{1}{2} \mathcal{M}_{6}-\frac{1}{2} m_{2} \mathcal{M}_{7} \\
& -\frac{1}{4}\left(m_{1}+m_{2}\right) \mathcal{M}_{10}-\frac{1}{4} m_{1} \mathcal{M}_{11}+\frac{1}{4}\left(s+m_{1} m_{2}\right) \mathcal{M}_{12} \\
& +\frac{1}{4}\left(m_{1}+m_{2}\right) \mathcal{M}_{13}+\frac{1}{4} m_{1} \mathcal{M}_{14}-\frac{1}{4}\left(s+m_{1} m_{2}\right) \mathcal{M}_{15} \\
B_{5} & =-\mathcal{M}_{1}+\left(m_{2}-m_{1}\right) \mathcal{M}_{5}-m_{1} \mathcal{M}_{6} \\
& -\left(s-m_{1} m_{2}\right) \mathcal{M}_{7}, \\
B_{6} & =-\mathcal{M}_{10}-\mathcal{M}_{11}+m_{2} \mathcal{M}_{12} \\
& -\mathcal{M}_{13}-\mathcal{M}_{14}+m_{2} \mathcal{M}_{15} \\
B_{7} & =-\frac{1}{2} \mathcal{M}_{10}-\frac{1}{2} \mathcal{M}_{11}+\frac{1}{2} m_{2} \mathcal{M}_{12} \\
& +\frac{1}{2} \mathcal{M}_{13}+\frac{1}{2} \mathcal{M}_{14}-\frac{1}{2} m_{2} \mathcal{M}_{15}, \\
B_{8} & =-\mathcal{M}_{8}-\mathcal{M}_{9}-\frac{1}{2} \mathcal{M}_{10}-\frac{1}{2} \mathcal{M}_{11}+\frac{1}{2} m_{2} \mathcal{M}_{12} \\
& -\frac{1}{2} \mathcal{M}_{13}-\frac{1}{2} \mathcal{M}_{14}+\frac{1}{2} m_{2} \mathcal{M}_{15} . \\
&
\end{aligned}
$$

For a gauge invariant amplitude, the following relations for the $B_{i}$ hold:

$$
\begin{aligned}
k^{2} B_{1}+2(k \cdot P) B_{2}+2(k \cdot q) B_{3}+2 k^{2} B_{4} & =0, \\
B_{5}+(k \cdot P) B_{6}+k^{2} B_{7}+(k \cdot q) B_{8} & =0 .
\end{aligned}
$$

Given these relations, one can eliminate two of the $B_{i}$ (conventionally, one takes $B_{3}$ and $B_{5}$ ) and rewrite the amplitude in a manifestly gauge invariant form:

$$
T_{f i}=i \bar{u}_{2} \sum_{i=1}^{6} A_{i} M_{i} u_{1}
$$

with the operator structures

$$
\begin{aligned}
& M_{1}=\frac{1}{2} \gamma_{5} \gamma_{\mu} \gamma_{\nu} F^{\mu \nu}, \\
& M_{2}=2 \gamma_{5} P_{\mu}\left(q-\frac{1}{2} k\right)_{\nu} F^{\mu \nu}, \\
& M_{3}=\gamma_{5} \gamma_{\mu} q_{\nu} F^{\mu \nu}, \\
& M_{4}=2 \gamma_{5} \gamma_{\mu} P_{\nu} F^{\mu \nu}-\left(m_{1}+m_{2}\right) M_{1}, \\
& M_{5}=\gamma_{5} k_{\mu} q_{\nu} F^{\mu \nu}, \\
& M_{6}=\gamma_{5} k_{\mu} \gamma_{\nu} F^{\mu \nu},
\end{aligned}
$$

where $F^{\mu \nu}=\epsilon^{\mu} k^{\nu}-\epsilon^{\nu} k^{\mu}$. The particular form of $M_{4}$ has been chosen such that

$$
i \bar{u}_{2} M_{4} u_{1} \rightarrow 2 \sqrt{m_{1} m_{2}} \mathbf{q} \cdot(\mathbf{k} \times \boldsymbol{\epsilon})
$$

in the nonrelativistic limit, where the baryon masses are large compared to the meson masses and three-momenta.

The $A_{i}$ are related to the $B_{i}$ via

$$
\begin{aligned}
A_{1}= & B_{1}-\frac{1}{2}\left(m_{1}+m_{2}\right) B_{6}, \\
A_{2}= & \frac{2}{M_{\phi}^{2}-t} B_{2}, \\
A_{3}= & -B_{8}, \\
A_{4}= & -\frac{1}{2} B_{6}, \\
A_{5}= & \frac{2}{s+u-m_{1}^{2}-m_{2}^{2}} \\
& \times\left(B_{1}-\frac{s-u+m_{2}^{2}-m_{1}^{2}}{2\left(M_{\phi}^{2}-t\right)} B_{2}+2 B_{4}\right), \\
A_{6}= & B_{7} .
\end{aligned}
$$

Following the conventions of Chew, Goldberger, Low and Nambu (CGLN) [49], and Berends, Donnachie and Weaver [50], we rewrite this once more, making use of the standard representation of spinors and Gamma matrices. In terms of Pauli spinors and matrices, one obtains

$$
\frac{1}{8 \pi \sqrt{s}} i \bar{u}_{2} \sum_{i=1}^{6} A_{i} M_{i} u_{1}=\chi_{2}^{\dagger} \mathbf{F} \chi_{1},
$$

where the matrix $\mathbf{F}$ reads

$$
\begin{aligned}
\mathbf{F}= & i \sigma \cdot \mathbf{b} \mathcal{F}_{1}+\sigma \cdot \hat{\mathbf{q}} \sigma \cdot(\hat{\mathbf{k}} \times \mathbf{b}) \mathcal{F}_{2}+i \sigma \cdot \hat{\mathbf{k}} \hat{\mathbf{q}} \cdot \mathbf{b} \mathcal{F}_{3} \\
& +i \sigma \cdot \hat{\mathbf{q}} \hat{\mathbf{q}} \cdot \mathbf{b} \mathcal{F}_{4}-i \sigma \cdot \hat{\mathbf{q}} b_{0} \mathcal{F}_{7}-i \sigma \cdot \hat{\mathbf{k}} b_{0} \mathcal{F}_{8} .
\end{aligned}
$$

Here, the hat over the three-vectors of course means normalization to a unit vector, and the four-vector $b_{\mu}$ is defined as

$$
b_{\mu}=\epsilon_{\mu}-\frac{\boldsymbol{\epsilon} \cdot \hat{\mathbf{k}}}{|\mathbf{k}|} k_{\mu} .
$$


By substituting the standard representation of the Dirac spinors and matrices on the left-hand side of eq. (D.4), one finds the following expressions for the so-called CGLNamplitudes $\mathcal{F}_{i}$ :

$$
\begin{aligned}
& \mathcal{F}_{1}=\left(\sqrt{s}-m_{1}\right) \frac{N_{1} N_{2}}{8 \pi \sqrt{s}}\left[A_{1}+\frac{k \cdot q}{\sqrt{s}-m_{1}} A_{3}\right. \\
& \left.+\left(\sqrt{s}-m_{2}-\frac{k \cdot q}{\sqrt{s}-m_{1}}\right) A_{4}-\frac{k^{2}}{\sqrt{s}-m_{1}} A_{6}\right], \\
& \mathcal{F}_{2}=\left(\sqrt{s}+m_{1}\right) \frac{N_{1} N_{2}}{8 \pi \sqrt{s}} \frac{|\mathbf{q}||\mathbf{k}|}{\left(E_{1}+m_{1}\right)\left(E_{2}+m_{2}\right)} \\
& \times\left[-A_{1}+\frac{k \cdot q}{\sqrt{s}+m_{1}} A_{3}\right. \\
& \left.+\left(\sqrt{s}+m_{2}-\frac{k \cdot q}{\sqrt{s}+m_{1}}\right) A_{4}-\frac{k^{2}}{\sqrt{s}+m_{1}} A_{6}\right], \\
& \mathcal{F}_{3}=\left(\sqrt{s}+m_{1}\right) \frac{N_{1} N_{2}}{8 \pi \sqrt{s}} \frac{|\mathbf{q}||\mathbf{k}|}{E_{1}+m_{1}} \\
& \times\left[\frac{m_{1}^{2}-s+\frac{1}{2} k^{2}}{\sqrt{s}+m_{1}} A_{2}+A_{3}-A_{4}-\frac{k^{2}}{\sqrt{s}+m_{1}} A_{5}\right], \\
& \mathcal{F}_{4}=\left(\sqrt{s}-m_{1}\right) \frac{N_{1} N_{2}}{8 \pi \sqrt{s}} \frac{|\mathbf{q}|^{2}}{E_{2}+m_{2}} \\
& \times\left[\frac{s-m_{1}^{2}-\frac{1}{2} k^{2}}{\sqrt{s}-m_{1}} A_{2}+A_{3}-A_{4}+\frac{k^{2}}{\sqrt{s}-m_{1}} A_{5}\right], \\
& \mathcal{F}_{7}=\frac{N_{1} N_{2}}{8 \pi \sqrt{s}} \frac{|\mathbf{q}|}{E_{2}+m_{2}}\left[\left(m_{1}-E_{1}\right) A_{1}\right. \\
& -\left(\frac{|\mathbf{k}|^{2}}{2 k_{0}}\left(2 k_{0} \sqrt{s}-3 k \cdot q\right)\right. \\
& \left.-\frac{\mathbf{q} \cdot \mathbf{k}}{2 k_{0}}\left(2 s-2 m_{1}^{2}-k^{2}\right)\right) A_{2} \\
& +\left(q_{0}\left(\sqrt{s}-m_{1}\right)-k \cdot q\right) A_{3} \\
& +\left(k \cdot q-q_{0}\left(\sqrt{s}-m_{1}\right)+\left(E_{1}-m_{1}\right)\left(\sqrt{s}+m_{2}\right)\right) A_{4} \\
& \left.+\left(q_{0} k^{2}-k_{0} k \cdot q\right) A_{5}-\left(E_{1}-m_{1}\right)\left(\sqrt{s}+m_{1}\right) A_{6}\right] \text {, } \\
& \mathcal{F}_{8}=\frac{N_{1} N_{2}}{8 \pi \sqrt{s}} \frac{|\mathbf{k}|}{E_{1}+m_{1}}\left[\left(E_{1}+m_{1}\right) A_{1}\right. \\
& +\left(\frac{|\mathbf{k}|^{2}}{2 k_{0}}\left(2 k_{0} \sqrt{s}-3 k \cdot q\right)\right. \\
& \left.-\frac{\mathbf{q} \cdot \mathbf{k}}{2 k_{0}}\left(2 s-2 m_{1}^{2}-k^{2}\right)\right) A_{2} \\
& +\left(q_{0}\left(\sqrt{s}+m_{1}\right)-k \cdot q\right) A_{3} \\
& +\left(k \cdot q-q_{0}\left(\sqrt{s}+m_{1}\right)+\left(E_{1}+m_{1}\right)\left(\sqrt{s}-m_{2}\right)\right) A_{4} \\
& \left.-\left(q_{0} k^{2}-k_{0} k \cdot q\right) A_{5}-\left(E_{1}+m_{1}\right)\left(\sqrt{s}-m_{1}\right) A_{6}\right] \text {, }
\end{aligned}
$$

where

$$
N_{i}=\sqrt{E_{i}+m_{i}}, \quad E_{i}=\sqrt{\mathbf{p}_{i}^{2}+m_{i}^{2}}, \quad i=1,2 .
$$

We remark that, starting from eq. (D.3), we have utilized the Lorentz condition $k \cdot \epsilon=0$. This is also valid when electroproduction is considered, since in that case the object $\epsilon^{\mu}$ is proportional to the electron-photon vertex of QED, and the condition then follows from current conservation.

Restricting ourselves to s-and p-waves, we can use the CGLN-amplitudes to arrive at the multipoles $E_{0+}, M_{1+}$, etc.:

$$
\left(\begin{array}{c}
E_{0+} \\
M_{1+} \\
M_{1-} \\
E_{1+}
\end{array}\right)=\int_{-1}^{1} d z\left(\begin{array}{cccc}
\frac{1}{2} P_{0} & -\frac{1}{2} P_{1} & 0 & \frac{1}{6} P_{0,2} \\
\frac{1}{4} P_{1} & -\frac{1}{4} P_{2} & -\frac{1}{12} P_{0,2} & 0 \\
-\frac{1}{2} P_{1} & \frac{1}{2} P_{0} & \frac{1}{6} P_{0,2} & 0 \\
\frac{1}{4} P_{1} & -\frac{1}{4} P_{2} & \frac{1}{12} P_{0,2} & \frac{1}{10} P_{1,3}
\end{array}\right)\left(\begin{array}{l}
\mathcal{F}_{1} \\
\mathcal{F}_{2} \\
\mathcal{F}_{3} \\
\mathcal{F}_{4}
\end{array}\right)
$$

and

$$
\left(\begin{array}{l}
L_{0+} \\
L_{1+} \\
L_{1-}
\end{array}\right)=\frac{k_{0}}{|\mathbf{k}|} \int_{-1}^{1} d z\left(\begin{array}{ccc}
\frac{1}{2} P_{1} & \frac{1}{2} P_{0} \\
\frac{1}{4} P_{2} & \frac{1}{4} P_{1} \\
\frac{1}{2} P_{0} & \frac{1}{2} P_{1}
\end{array}\right)\left(\begin{array}{l}
\mathcal{F}_{7} \\
\mathcal{F}_{8}
\end{array}\right),
$$

where $P_{\ell} \equiv P_{\ell}(z)$ are the usual Legendre polynomials and $z$ is $\hat{\mathbf{q}} \cdot \hat{\mathbf{k}}$, i.e. the cosine of the scattering angle in the c.m. frame. Furthermore, the abbreviations

$$
P_{0,2}=P_{0}-P_{2} \quad \text { and } \quad P_{1,3}=P_{1}-P_{3}
$$

were used.

A word on units: Since we use

$$
\hbar=c=1, \quad e^{2}=4 \pi \alpha
$$

and normalize our Dirac spinors like $\bar{u} u=2 m$, the invariant amplitude $B_{5}$ has dimension $\mathrm{GeV}^{-1}$, as can be seen, e.g., from the contribution of the graph corresponding to the 'Kroll-Ruderman' term. Therefore, the scattering amplitude $T_{f i}$ is dimensionless (see eq. (D.1) $)$, while the CGLN-amplitudes as well as the multipoles have dimension $\mathrm{GeV}^{-1}$.

The unpolarized differential cross section for $\gamma p \rightarrow$ $\mathrm{BM}$ is given in terms of the CGLN-amplitudes as [50]

$$
\begin{aligned}
\frac{d \sigma}{d \Omega} & =\frac{|\mathbf{q}|}{|\mathbf{k}|}\left\{\left|\mathcal{F}_{1}\right|^{2}+\left|\mathcal{F}_{2}\right|^{2}+\frac{1}{2}\left|\mathcal{F}_{3}\right|^{2}+\frac{1}{2}\left|\mathcal{F}_{4}\right|^{2}\right. \\
& +\operatorname{Re}\left(\mathcal{F}_{1} \mathcal{F}_{4}^{*}\right)+\operatorname{Re}\left(\mathcal{F}_{2} \mathcal{F}_{3}^{*}\right) \\
& +\left(\operatorname{Re}\left(\mathcal{F}_{3} \mathcal{F}_{4}^{*}\right)-2 \operatorname{Re}\left(\mathcal{F}_{1} \mathcal{F}_{2}^{*}\right)\right) \cos \theta \\
& -\left(\frac{1}{2}\left|\mathcal{F}_{3}\right|^{2}+\frac{1}{2}\left|\mathcal{F}_{4}\right|^{2}+\operatorname{Re}\left(\mathcal{F}_{1} \mathcal{F}_{4}^{*}+\mathcal{F}_{2} \mathcal{F}_{3}^{*}\right)\right) \cos ^{2} \theta \\
& \left.-\operatorname{Re}\left(\mathcal{F}_{3} \mathcal{F}_{4}^{*}\right) \cos ^{3} \theta\right\} .
\end{aligned}
$$




\section{References}

1. S. Capstick and N. Isgur, Phys. Rev. D 34 (1986) 2809.

2. L. Y. Glozman, W. Plessas, K. Varga and R. F. Wagenbrunn, Phys. Rev. D 58 (1998) 094030 arXiv:hep-ph/9706507.

3. U. Löring, K. Kretzschmar, B. C. Metsch and H. R. Petry, Eur. Phys. J. A 10 (2001) 309 arXiv:hep-ph/0103287.

4. K. H. Glander et al., Eur. Phys. J. A 19 (2004) 251 arXiv:nucl-ex/0308025.

5. R. Lawall et al., Eur. Phys. J. A 24 (2005) 275 arXiv:nucl-ex/0504014.

6. J. W. C. McNabb et al. [CLAS Collaboration], Phys. Rev. C 69 (2004) 042201 arXiv:nucl-ex/0305028.

7. R. Bradford et al. [CLAS Collaboration], Phys. Rev. C $\mathbf{7 3}$ (2006) 035202 arXiv:nucl-ex/0509033.

8. R. Castelijns et al. [CBELSA/TAPS Collaboration], arXiv:nucl-ex/0702033

9. R. G. T. Zegers et al. [LEPS Collaboration], Phys. Rev. Lett. 91 (2003) 092001 arXiv:nucl-ex/0302005.

10. A. V. Anisovich, A. Sarantsev, O. Bartholomy, E. Klempt, V. A. Nikonov and U. Thoma, Eur. Phys. J. A 25 (2005) 427 arXiv:hep-ex/0506010.

11. A. V. Sarantsev, V. A. Nikonov, A. V. Anisovich, E. Klempt and U. Thoma, Eur. Phys. J. A 25 (2005) 441 arXiv:hep-ex/0506011.

12. A. Usov and O. Scholten, Phys. Rev. C 72 (2005) 025205 arXiv:nucl-th/0503013.

13. C. E. Carlson and J. L. Poor, Phys. Rev. D 38 (1988) 2758.

14. D. B. Leinweber, T. Draper and R. M. Woloshyn, Phys. Rev. D 46 (1992) 3067 arXiv:hep-lat/9208025.

15. N. Kaiser, P. B. Siegel and W. Weise, Nucl. Phys. A 594 (1995) 325 arXiv:nucl-th/9505043.

16. N. Kaiser, P. B. Siegel and W. Weise, Phys. Lett. B 362 (1995) 23 arXiv:nucl-th/9507036.

17. E. Oset and A. Ramos, Nucl. Phys. A 635 (1998) 99 arXiv:nucl-th/9711022.

18. J. A. Oller, E. Oset and A. Ramos, Prog. Part. Nucl. Phys. 45 (2000) 157 arXiv:hep-ph/0002193.

19. J. A. Oller and U.-G. Meißner, Phys. Lett. B 500 (2001) 263 arXiv:hep-ph/0011146.

20. M. F. M. Lutz and E. E. Kolomeitsev, Nucl. Phys. A 700 (2002) 193 arXiv:nucl-th/0105042.

21. N. Kaiser, T. Waas and W. Weise, Nucl. Phys. A 612, 297 (1997) arXiv:hep-ph/9607459.

22. J. Caro Ramon, N. Kaiser, S. Wetzel and W. Weise, Nucl. Phys. A 672 (2000) 249 arXiv:nucl-th/9912053.

23. J. C. Nacher, E. Oset, H. Toki and A. Ramos, Phys. Lett. B 455 (1999) 55 arXiv:nucl-th/9812055.

24. B. Borasoy, E. Marco and S. Wetzel, Phys. Rev. C 66 (2002) 055208 arXiv:hep-ph/0212256.

25. B. Borasoy, P. C. Bruns, U.-G. Meißner and R. Nißler, Phys. Rev. C 72 (2005) 065201 arXiv:hep-ph/0508307.

26. A. N. Kvinikhidze and B. Blankleider, Phys. Rev. C 60 (1999) 044003 arXiv:nucl-th/9901001.

27. J. A. Oller and E. Oset, Nucl. Phys. A 620 (1997) 438 [Erratum-ibid. A 652 (1999) 407] arXiv:hep-ph/9702314].

28. J. Nieves and E. Ruiz Arriola, Nucl. Phys. A 679 (2000) 57 arXiv:hep-ph/9907469.

29. F. E. Close and R. G. Roberts, Phys. Lett. B 316 (1993) 165 arXiv:hep-ph/9306289; B. Borasoy, Phys. Rev. D 59 (1999) 054021 arXiv:hep-ph/9811411].
30. C. Bennhold, T. Mart and D. Kusno, arXiv:nucl-th/9703004

31. B. Ananthanarayan, G. Colangelo, J. Gasser and H. Leutwyler, Phys. Rept. 353 (2001) 207 arXiv:hep-ph/0005297.

32. P. Buettiker, S. Descotes-Genon and B. Moussallam, Eur. Phys. J. C 33 (2004) 409 arXiv:hep-ph/0310283.

33. T. Becher and H. Leutwyler, JHEP 0106 (2001) 017 arXiv:hep-ph/0103263.

34. T. M. Knasel et al., Phys. Rev. D 11 (1975) 1.

35. R. D. Baker et al., Nucl. Phys. B 141 (1978) 29.

36. U.-G. Meißner and J. A. Oller, Nucl. Phys. A 673 (2000) 311 arXiv:nucl-th/9912026.

37. B. Borasoy, U.-G. Meißner and R. Nißler, Phys. Rev. C 74 (2006) 055201 arXiv:hep-ph/0606108.

38. R. D. Baker et al., Nucl. Phys. B 145 (1978) 402.

39. A. Baldini, V. Flaminio, W. G. Moorhead, and D. R. O. Morrison, in: Landolt-Börnstein, Vol. 12a, ed. H. Schopper (Springer, Berlin, 1988).

40. A. V. Anisovich, V. Kleber, E. Klempt, V. A. Nikonov, A. V. Sarantsev and U. Thoma, arXiv:0707.3596 [hep-ph].

41. T. Inoue, E. Oset and M. J. Vicente Vacas, Phys. Rev. C 65 (2002) 035204 arXiv:hep-ph/0110333.

42. A. Motzke, C. Elster and C. Hanhart, Phys. Rev. C 66 (2002) 054002 arXiv:nucl-th/0207047.

43. M. Frink and U.-G. Meißner, JHEP 0407 (2004) 028 arXiv:hep-lat/0404018.

44. J. A. Oller, Eur. Phys. J. A 28 (2006) 63 arXiv:hep-ph/0603134.

45. N. Beisert, Diploma thesis, TU München (2001), unpublished.

46. D. Djukanovic, J. Gegelia and S. Scherer, Eur. Phys. J. A 29 (2006) 337 arXiv:hep-ph/0604164.

47. G. 't Hooft and M. J. G. Veltman, Nucl. Phys. B 153 (1979) 365.

48. R. Mertig, M. Bohm and A. Denner, Comput. Phys. Commun. 64 (1991) 345. http://www.feyncalc.org

49. G. F. Chew, M. L. Goldberger, F. E. Low and Y. Nambu, Phys. Rev. 106 (1957) 1345.

50. F. A. Berends, A. Donnachie and D. L. Weaver, Nucl. Phys. B 4 (1967) 1. 